\title{
Variational data assimilation for transient blood flow simulations - Cerebral aneurysms as an illustrative example
}

\author{
S. W. Funke and M. Nordaas and Ø. Evju and M. S. Alnæs and K.-A. Mardal \\ Simula Research Laboratory, Norway and Chalmers University of Technology, Sweden and Department of \\ Mathematics, University of Oslo, Norway
}

\begin{abstract}
SUMMARY
Several cardiovascular diseases are caused from localised abnormal blood flow such as in the case of stenosis or aneurysms. Prevailing theories propose that the development is caused by abnormal wall-shear stress in focused areas. Computational fluid mechanics have arisen as a promising tool for a more precise and quantitative analysis, in particular because the anatomy is often readily available even by standard imaging techniques such as magnetic resonance and computed tomography angiography. However, computational fluid mechanics rely on accurate initial and boundary conditions which are difficult to obtain. In this paper we address the problem of recovering high resolution information from noisy and low-resolution physical measurements of blood flow (for example from PC-MRI) using variational data assimilation based on a transient Navier-Stokes model. Numerical experiments are performed in both 3D (2D space and time) and 4D (3D space and time) and with pulsatile flow relevant for physiological flow in cerebral aneurysms. The results demonstrate that, with suitable regularisation, the model accurately reconstructs flow, even in the presence of significant noise. Copyright (C) 0000 John Wiley \& Sons, Ltd.
\end{abstract}

Received ...

KEY WORDS: blood flow, variational data assimilation, optimal control, finite element method, adjoint equations, Navier-Stokes

\section{INTRODUCTION}

Computational simulations of blood flow have the potential to assist clinical decisions, for example when evaluating the risk of rupture of an aneurysm $[1,2,3]$. In contrast to physical measurement techniques, computational fluid dynamics (CFD) allows for nearly arbitrary high resolution. Further, CFD allows the computation of non-observable variables, such as the blood pressure and wall-shear stresses, both of which are considered important factors in vascular diseases [4, 5, 6, 3]. However, a major challenge of current CFD models that the validity and accuracy of their results depend on the underlying model assumptions, the model parameters [7, 8], the boundary conditions $[9,10]$, and the segmentation of the vascular geometry $[11,12,13]$. These parameters are typically specific to each patient $[10,14]$ and partially or fully unknown.

One reason for the lack of sufficiently good patient-specific data is that the resolution of noninvasive measurement techniques, such as ultrasound or phase-contrast magnetic resonance imaging (PC-MRI), is coarse compared to CFD, and their accuracy limited by the presence of noise. For instance, PC-MRI provides 4D (3D space and time) images of velocity at a spatial resolution between $0.3-0.7 \mathrm{~mm}$ with a uniform voxel size $[15,16]$ whereas the normal resolution in CFD is $0.1-0.2 \mathrm{~mm}$, c.f. e.g. [1, 17], and where the need for even finer CFD resolution has been advocated $[18,19]$. The temporal resolution of PC-MRI is typically limited to around 30 samples

${ }^{*}$ Correspondence to: Simula Research Laboratory, P.O. Box 134, 1325 Lysaker, Norway 
per heart beat. Furthermore, PC-MRI acquisitions may last 5-10 minutes, and the data are then phase-averaged over several hundred cardiac cycles in order to reduce to signal-to-noise ratio. In contrast, the temporal resolution in CFD simulations is typically on the order of thousands of time steps per second. As a result, there is often a notable discrepancy between CFD simulations and PCMRI measurements [16, 15, 20]. To what extent these discrepancies arise from modelling errors, the spatio-temporal averaging, or measurement errors is at present difficult to assess in particular because of the methodological challenge of incorporating the available data and control parameters in a simulation framework.

A promising remedy is hence to combine CFD and 4D MRI using data assimilation. The CFD would then potentially add resolution and reduce noise. The idea of applying data assimilating techniques to blood flow models has received significant attention in recent years, see [21] for an overview. In particular variational data assimilation, which identifies unknown model parameters such that the difference between physical observations and model results are minimised, has been studied in the general setting for the optimal control of the Navier-Stokes equations [22, 23, 24] and in the specific case of blood-flow simulations [25, 26, 27, 28, 29]. The mathematical theory behind variational data assimilation is partially developed, in particular the well-possessedness of the (regularized) inverse minimization problem for both flow and fluid-structure interaction problem has been addressed in, e.g., [24, 30]. Alternative, more advanced data assimilation strategies use reduced basis methods and/or Bayesian parameter estimating, c.f. e.g., [31, 32, 33, 34]. For bifurcation problems, more general strategies of setting boundary conditions have been developed in $[35,36,37]$, in particular since the main problem in these cases is the determination of the flow division rather than a complete set of boundary conditions as long as flow extensions are appropriate.

The benefits of variational data assimilation comes at a price. The adjoint model of the NavierStokes equations must be derived and implemented, which is difficult, especially for transient flow models. Further, the computational complexity is between 50-100 times higher than one model simulation. This presents a problem especially for 4D (3D space and time) models. As a consequence, most numerical studies on variational data assimilation for blood flow currently assume steady-state flow and/or 2D geometries - assumptions that clearly need to be overcome for realistic studies.

The goal of this paper is to derive and implement variational data assimilation for transient hemodynamics, and to investigate its feasibility and robustness on cerebral aneurysms with coarse and noisy velocity measurements. Specifically, we consider an idealized 3D case (2D space and time) and a 4D case (3D space and time) with PC-MRI 4D velocity data. The unknown model parameters, to be identified by the data assimilation, are the initial and boundary conditions of the model equations. This, and many other of our modelling choices are too simplistic to be used in real hemodynamic studies, but the present study is a necessary and important step towards a full assimilated blood flow model - in particular because the proposed methodology generalises to more advanced setups, such as inclusion of periodic boundary conditions, and the assimilation of other model parameters such as the viscosity and even the geometry through shape optimisation [38]. Our results show that the assimilation procedure is computational expensive, but yields surprisingly robust and accurate results even for sparse and noisy measurements.

The remainder of the paper is as follows. In Section 2, we formulate the variational dataassimilation as a mathematical optimisation problem constrained by the Navier-Stokes equations. Special considerations will be put on the spatial and temporal regularisation, and the inclusion of data that are coarse with respect to the time resolution. The latter is important because the number of samples per cardiac cycle is typically in the order 20-40 while the number of timesteps in a CFD simulation typically is $100-10,000$. Section 3 presents the numerical details. In short, these are based on the finite element method, the reduced optimisation problem and a discretise-thenoptimise approach. Section 4 presents numerical experiments of blood flow through aneurysms. First, an idealistic 3D example (2D space and time) is used to verify the assimilation procedure, and to test its robustness against noisy data, the regularisation amplitude, the sparsity in the physical observations and the choice of the controlled boundaries. Second, a 4D case (3D space and time) is presented, based on 4D data obtained from a PC-MRI scan of an arterial bifurcation with aneurysm 


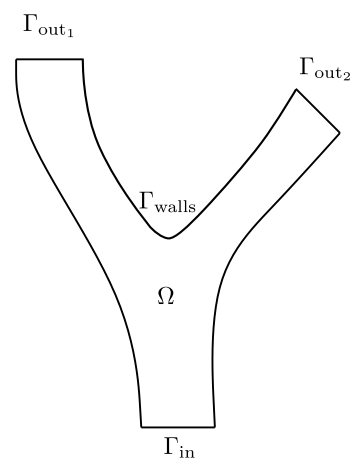

Figure 1. The model scenario considered in this paper: a small subset of the artery system with one inlet and two outlet boundaries in 2D and 3D.

in a dog. This example shows the feasibility of 4D data assimilation for blood flow problems, and is used to qualitatively compare variational data assimilation with a "traditional" blood flow simulation approach where the boundary conditions are interpolated directly from the physical measurements.

\section{MATHEMATICAL FORMULATION}

\subsection{Blood flow model}

Computational modelling in cerebral aneurysm studies often assume Newtonian flow with rigid walls, which appear to be adequate $[39,40]$. Therefore, we model the blood flow through a vessel with the incompressible Navier-Stokes equations

$$
\begin{array}{rlrl}
u_{t}+(u \cdot \nabla) u-\nu \Delta u+\nabla p & =f & & \text { in } \Omega \times(0, T], \\
\nabla \cdot u=0 & & \text { in } \Omega \times(0, T] .
\end{array}
$$

Here, $\Omega \times(0, T]$ is the space-time domain, $u$ and $p$ are the blood velocity and (scaled) pressure fields, $\nu$ is the (kinematic) viscosity and $f$ describes external body forces. A more complete blood flow model could for example incorporate non-Newtonian effects [41] or the fluid structure interactions between the blood and the vessel wall [?]. However, for the purpose of this paper it is sufficient to consider (1) and to note that the proposed techniques also apply to more complex models.

We only model a small subset of the artery system and the boundaries of the computational domain consists of a physical boundary, the vessel walls, as well non-physical boundaries at inlets and outlets. Again for simplicity, we consider the common scenario of one inlet and two outlets as sketched in figure 1 . To close the system, we specify suitable initial and boundary conditions

$$
\begin{aligned}
u & =u_{0}, & & \text { on } \Omega \times\{t=0\}, \\
u & =g_{D}, & & \text { on } \Gamma_{D} \times(0, T], \\
p n-\mu \partial_{n} u & =0, & & \text { on } \Gamma_{\text {out }_{2}} \times(0, T], \\
u & =0, & & \text { on } \Gamma_{\text {walls }} \times(0, T],
\end{aligned}
$$

with initial velocity $u_{0}$ and Dirichlet boundary value $g_{D}$ on the boundary $\Gamma_{D}:=\Gamma_{\text {in }} \cup \Gamma_{\text {out }_{1}}$. The normal vector is denoted as $n$. A traction free boundary is assumed on the outlet $\Gamma_{\text {out }_{2}}$, see (2c), which implies that the vessel is straight in the surroundings of this outlet. If this assumption is not valid, other choices for (2c) are possible, but mass conservation must be ensured, i.e. that $\int_{\Gamma} u \cdot n \mathrm{~d} x=0$.

For data-assimilation based on PC-MRI measurement it is more appropriate to replace the initial condition by a time-periodic condition. From a practical point of view, however, the implementation of such a condition introduces further challenges in the implementation and we therefore resort to a forward problem defined in terms of an initial condition. 


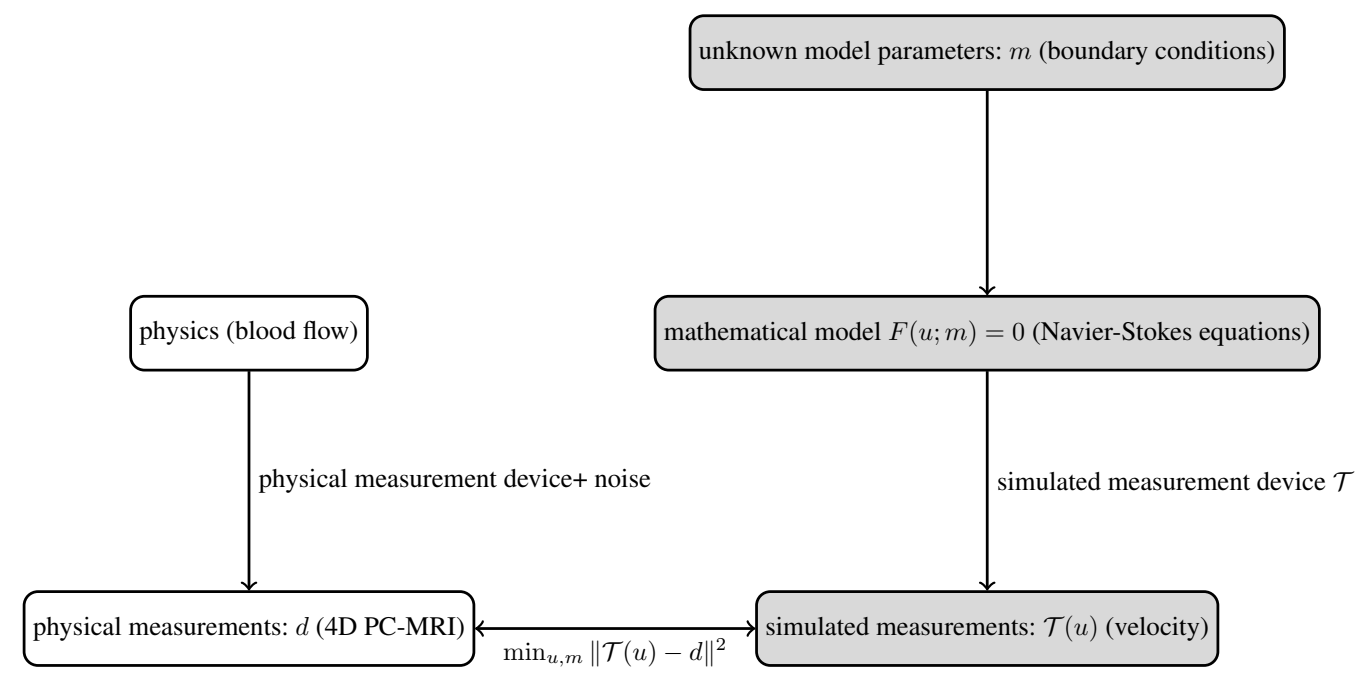

Figure 2. Variational data assimilation replicates the physical measurement acquisition (white boxes) in a mathematical model (grey boxes) and minimises the discrepancy between measured and simulated measurements by varying the unknown parameters of the model.

\subsection{Variational data assimilation}

Variational data assimilation is a mathematical technique to recover unknown model parameters from physical measurements by utilizing an optimal control approach. It was introduced in the context of metereological models [42], but has since then been applied to other areas such as oceanography [43] and mantle convection [44]. For brevity we only give a high-level overview of variational data assimilation. For a thorough introduction we refer the reader to [45].

Figure 2 visualises the concept of variational data assimilation. The available measurements of the physical system are denoted as $d$. Variational data assimilation requires mathematical models that replicate both the physical processes and the measurement acquisition. The physical processes are typically described by a partial differential equation. We denote this PDE as $F(u ; m)=0$, where $u$ is the solution and $m$ are unknown parameters, such as the initial and boundary conditions. Similarly, we can build a model for the physical measurement acquisition, which computes 'virtual measurements' from the solution $u$. We denote this operation as $\mathcal{T}(u)$. The core idea of variational data assimilation is now to determine the unknown parameters $m$ such that the discrepancy between simulated and physical measurements is minimised:

$$
\begin{gathered}
\min _{u, m}\|\mathcal{T}(u)-d\|^{2} \\
\text { subject to } \quad F(u ; m)=0 .
\end{gathered}
$$

The solution $u$ of (3) can be used to extract previously unknown data.

For the variational data assimilation of blood flow, the aim is to recover the flow velocity and pressure fields from noisy physical velocity measurements with limited spatial and temporal resolution. The recovered fields should be physically reasonable, in the sense that they satisfy the mathematical model considered. Hence we use equations (1) and (2) as the mathematical model (4). For this model, the unknown parameters are the initial condition and the Dirichlet boundary, that is $m=\left(u_{0}, g_{D}\right)$. We assume that the available physical measurements are $N$ velocity fields on a subdomain $\Omega_{\mathrm{obs}} \subseteq \Omega$, that is $d=\left(d_{1}, \ldots, d_{N}\right) \in L^{2}\left(\Omega_{\mathrm{obs}}\right)^{N}$. For example, each observation might be an instantaneous snapshot of the blood flow velocity, or the average over a time interval. The observation subdomain $\Omega_{\mathrm{obs}}$ is introduced because the physical observations might be available 
only in parts of the computational domain. With that, the objective (3) becomes

$$
J(u):=\sum_{n=1}^{N} \int_{\Omega_{\mathrm{obs}}}\left|\mathcal{T}_{n} u-d_{n}\right|^{2} \mathrm{~d} x .
$$

Each observation operator $\mathcal{T}_{n}, n=1, \ldots, N$ models the acquisition of the n'th measurement. We consider two cases: in the first, the measurement device takes instantaneously measurements at $N$ timelevels $t_{1}, . ., t_{N}$. In this case, the observation operators are pointwise evaluations in time, that is $\mathcal{T}_{n} u(x)=u\left(x, t_{n}\right) \forall x$ in $\Omega_{\mathrm{obs}}$. In this second case, the measurement device takes averaged measurements over fixed time periods. Here, the observation operators are time averaged evaluations of the velocity state, that is $\mathcal{T}_{n} u(x)=\int_{t_{n-1}}^{t_{n}} u(x, t) \mathrm{d} t /\left(t_{n}-t_{n-1}\right) \forall x$ in $\Omega_{\text {obs }}$.

In summary, the variational data assimilation problem can be stated as a minimisation problem constrained by the Navier-Stokes equations:

$$
\min _{\substack{(u, p) \in Y \\\left(u_{0}, g_{D}\right) \in M}} J(u)+\mathcal{R}\left(u_{0}, g_{D}\right) \quad \text { subject to (1)-(2), }
$$

where $M$ and $Y$ are suitable function spaces, to be determined in section 3.3. Note that we also added a regularisation term $\mathcal{R}$, which is required to ensure well-posedeness of the problem, and needs to be chosen depending on the amount of physical observations [26]. In particular, [27] showed that a linearised variation of (6) is well-defined if sufficient physical observations are available. For the following numerical examples, we apply a Tikhonov regularisation:

$$
\mathcal{R}\left(u_{0}, g_{D}\right)=\frac{\alpha}{2}\left\|g_{D}\right\|_{\Gamma_{D} \times(0, T]}^{2}+\frac{\gamma}{2}\left\|u_{0}\right\|_{\Omega}^{2},
$$

where the coefficients $\alpha$ and $\gamma$ determine how strongly the problem is regularised in the given norms.

2.2.1. Choice of norms The choice of norms in the regularisation term (7) specifies the expected regularity of the reconstructed blood flow. For instance, [28] has shown that a unsuitable choice can have a negative impact on the quality of on the reconstructed data.

The norm used for the initial data is

$$
\left\|u_{0}\right\|_{\Omega}=\left\|u_{0}\right\|_{H^{1}(\Omega)}=\left(\int_{\Omega}\left|u_{0}\right|^{2}+\left|\nabla u_{0}\right|^{2} \mathrm{~d} x\right)^{\frac{1}{2}},
$$

and for the boundary

$$
\left\|g_{D}\right\|_{\Gamma_{D} \times(0, T]}=\left(\int_{0}^{T} \int_{\Omega}\left|g_{D}\right|^{2}+\left|\nabla g_{D}\right|^{2}+\left|\dot{g}_{D}\right|^{2}+\left|\nabla \dot{g}_{D}\right|^{2} \mathrm{~d} x \mathrm{~d} t\right)^{\frac{1}{2}} .
$$

We remark that the norms used require more smoothness on the boundary and initial data than is usually required for the variational formulation of the Navier-Stokes equations, in particular for the time derivative $\dot{g}_{D}$.

\section{NUMERICAL SOLUTION}

\subsection{Formulation of the reduced problem}

We solve the optimisation problem (6) in its reduced formulation. The idea of the reduced formulation is to eliminate the explicit PDE-constraints by implicitly enforcing the PDE in the functional. This strategy is common practice for solving PDE-constrained optimisation problems and described in more detail in [46].

The reduced problem of (6) is obtained by considering the velocity $u$ as an implicit function of the initial and boundary conditions by solving the Navier-Stokes equations (1) and (2). We 
denote this velocity operator as $u(m)$, where $m=\left(u_{0}, g_{D}\right) \in M$ denotes the controlled variables. The functional (5) can now be written in its reduced form

$$
\hat{J}(m)=J(u(m))+\mathcal{R}(m) .
$$

In words, the reduced functional $\hat{J}$ implicitly satisfies the Navier-Stokes equations. This means that an evaluation of $\hat{J}$ for a specific $m$ automatically invokes a Navier-Stokes solve to obtain the velocity $u(m)$, ensuring that (1) and (2) are always satisfies. The Navier-Stokes equations are therefore not required as explicit constraints to reduced functional $\hat{J}$. This bring us to the reduced optimisation problem

$$
\min _{m \in M} \hat{J}(m)
$$

In contrast to (6), the reduced problem is an unconstrained optimisation problem and can hence be solved with an unconstrained optimisation method.

\subsection{Optimisation}

The minimisation problem (9) is solved with the Broyden-Fletcher-Goldbarb-Shanno (BFGS) algorithm. This section presents a brief overview of the method in a function space setting, which is crucial to obtain discretisation-independent convergence, and its implementation.

BFGS solves the minimisation problems iteratively by generating a sequence of points $m_{0}, m_{1}, \ldots$, which approximate a minimiser of $\hat{J}$. In each iteration, evaluations of the derivative $D \hat{J}\left(m_{k}\right) \in M^{*}$ are used to determine a direction $d_{k} \in M$ in which the functional is decreasing. This general descent algorithm in Hilbert spaces is formulated in algorithm 1.

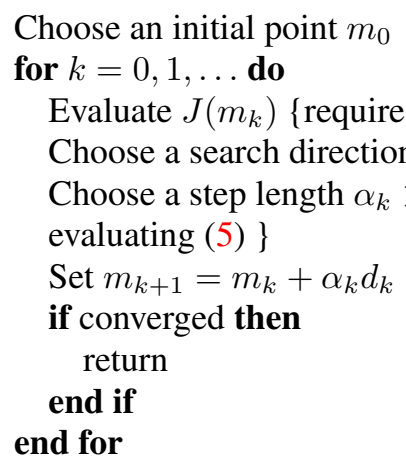

Algorithm 1: A general descent algorithm in Hilbert spaces, applied to the reduced minimisation problem (9).

The search direction $d_{k}=H_{k} D \hat{J}\left(m_{k}\right)$ is a descent direction if the operator $H_{k}: M^{*} \rightarrow M$ is positive definite and self-adjoint. The choice of operators $H_{k}$ mapping derivatives to search directions essentially characterises the method. For example, taking $H_{k}=H$ as the Riesz operator for $M$ (i.e. choosing $d_{k}$ to be the gradient of $\hat{J}$ at $m_{k}$ ) results in a steepest algorithm. Setting $H_{k}=D^{2} \hat{J}\left(m_{k}\right)^{-1}$, assuming $\hat{J}$ is convex, results in a Newton algorithm.

The BFGS algorithm is a descent method of quasi-Newton type, which have good convergence properties. Furthermore, they do not require evaluations of the Hessian, but instead maintain an iteratively constructed inverse Hessian approximation. The update formula specific to the BFGS algorithm is

$$
H_{k+1}=\left(1-\frac{s_{k+1} \otimes y_{k+1}}{\rho_{k+1}}\right) H_{k}\left(1-\frac{y_{k+1} \otimes s_{k+1}}{\rho_{k+1}}\right)+\frac{s_{k+1} \otimes s_{k+1}}{\rho_{k+1}}
$$


see e.g. [47, Chapter 6]. Here, $\otimes: X \times Y \rightarrow \mathcal{B}\left(Y^{*}, X\right)$ denotes the outer product defined by $(x \otimes y)(z)=x\langle z, y\rangle_{Y^{*}, Y}$, for $x \in X$ and $y \in Y$, where $\langle\cdot, \cdot\rangle_{Y^{*}, Y}$ denotes duality coupling, and

$$
\begin{aligned}
s_{k+1} & =m_{k+1}-m_{k}, \\
y_{k+1} & =D \hat{J}\left(m_{k+1}\right)-D \hat{J}\left(m_{k}\right), \\
\rho_{k+1} & =\left\langle y_{k+1}, s_{k}\right\rangle_{M^{*}, M} .
\end{aligned}
$$

Note that the initial $H_{0}: M^{*} \rightarrow M$ has to prescribed. A natural choice is to take $H_{0}$ to be Riesz operator for the space $M$. That is, $H_{0}$ is the unique operators such that

$$
\left(m_{0}, m_{1}\right)_{M}=\left\langle H_{0}^{-1} m_{0}, m_{1}\right\rangle_{M^{*}, M}
$$

for all $m_{0}, m_{1} \in M$. This definition of $H_{0}$ allows for mesh-independent convergence [48], and is readily seen to coincide with the second order partial derivative of $\hat{J}$ with respect to $M$. If $D^{2} \hat{J}(m)-H_{0}^{-1}$ is compact, the method converges superlinearly, see e.g. [49].

For practical implementations, it is common to truncate the update formula (10) and store only the last $3-10$ pairs of vectors $y_{k}$ and $s_{k}$. The step lengths $\alpha_{k}$ in algorithm 1 should chosen to satisfy the Wolfe conditions, which ensures the convergence of the method [47, chapter 6].

\subsection{Discretisation}

The optimisation method in section 3.2 requires evaluations of the reduced functional $\hat{J}(m)$ and its derivative $D \hat{J}(m)$. The former requires the numerical solution of the Navier-Stokes equations, which is described in section 3.3.1. The derivatives are computed by solving the adjoint equations as described in section 3.3.2.

3.3.1. Discretisation of the Navier-Stokes equations The Navier-Stokes equations are discretised with a $\theta$ time-stepping scheme and the finite element method. Other discretisation schemes, such as Runge-Kutta methods, may be employed if desired [50, 51, 52]. The controlled Dirichlet boundary conditions are weakly enforced with the Nitsche method [53]. An advantage of the Nitsche approach is that the boundary values are explicitly included in the variational formulation, which simplifies the automated derivation of the adjoint equations, which is exploited in our implementation.

For the spatial discretisation, we consider conforming finite element spaces

$$
\begin{aligned}
V_{h} & \subset H_{0, \Gamma_{\text {walls }}^{1}}^{1}(\Omega)=\left\{u \in H^{1}(\Omega):\left.u\right|_{\Gamma_{\text {walls }}}=0\right\} \\
Q_{h} & \subset L^{2}(\Omega) .
\end{aligned}
$$

For the time discretisation we assume a partition of the interval $[0, T]$ with a constant timestep $\delta t$. Applying a standard $\theta$ time-stepping scheme to the Navier-Stokes equations (1), we obtain a sequence of nonlinear problems: For $k=0, \ldots, N-1$, let $u^{k+\theta}=\theta u^{k+1}+(1-\theta) u^{k}$ and find $\left(u^{k+1}, p^{k+1}\right) \in V_{h} \times Q_{h}$ such that

$$
\begin{aligned}
\frac{u^{k+1}-u^{k}}{\delta t}-\nu \Delta u^{k+\theta}+\left(u^{k+\theta} \cdot \nabla\right) u^{k+\theta}-\nabla p^{k+1} & =0, \\
\nabla \cdot u^{k+1} & =0,
\end{aligned}
$$


subject to the boundary conditions (2). The equations (13) are integrated against test functions $v \in V_{h}$ and $q \in Q_{h}$ in order to obtain a nonlinear variational problem at each time $t_{k}$,

$$
\begin{aligned}
0=\int_{\Omega} & \left(\frac{u^{k+1}-u^{k}}{\delta t}\right) \cdot v+\nu \nabla u^{k+\theta}: \nabla v \mathrm{~d} x \\
& +\int_{\Omega}\left(u^{k+\theta} \cdot \nabla\right) u^{k+\theta} \cdot v \mathrm{~d} x \\
& +\int_{\Omega} q \nabla \cdot u^{k+1}+p^{k+1} \nabla \cdot v \mathrm{~d} x \\
& -\int_{\Gamma_{D}}\left(\nu \frac{\partial u^{k+\theta}}{\partial n}-p^{k+1} n\right) \cdot v \mathrm{~d} s \\
& -\int_{\Gamma_{D}}\left(\theta \nu \frac{\partial v}{\partial n}-q n\right) \cdot\left(u^{k+1}-g^{k+1}\right) \mathrm{d} s \\
& +\int_{\Gamma_{D}} \frac{\nu \sigma}{h}\left(u^{k+1}-g_{D}^{k+1}\right) \cdot v \mathrm{~d} s .
\end{aligned}
$$

The nonlinear variational problem (14) consists of a volume integral and a boundary integral over $\Gamma_{D}$. The volume integral coincides with the "standard" variational form of (13) obtained when the boundary condition (2b) is strongly imposed. The second, boundary integral part of the variational problem arises from the weakly imposing the Dirichlet boundary condition (17) with Nitsche's method, and is discussed in detail below.

The discrete spaces for the state and control variables are

$$
\begin{aligned}
& Y=V_{h}^{N} \times Q_{h}^{N} \\
& M=V_{h} \times\left(T_{\Gamma} V_{h}\right)^{N},
\end{aligned}
$$

and we introduce the notation

$$
\begin{aligned}
y & =(u, p) \in Y, \\
u & =\left(u_{1}, \ldots, v_{N}\right) \in V_{h}^{N} \\
p & =\left(p_{1}, \ldots, q_{N}\right) \in Q_{h}^{N} \\
m & =\left(u_{0}, g_{1}, \ldots, g_{N}\right) \in M
\end{aligned}
$$

The sequence of variational problems (14) is reformulated as an operator equation combining all the timesteps,

$$
\mathcal{F}(m, y)=\sum_{k=0}^{N-1}\left\{\mathcal{F}_{k, \Omega}(m, y)+\mathcal{F}_{k, \Gamma_{D}}(m, y)\right\}=0,
$$

where $\mathcal{F}_{k, \Omega}: Y \rightarrow Y^{*}$ is the operator combining all the volume integrals in (14), i.e.

$$
\begin{aligned}
\mathcal{F}_{k, \Omega}(m, y ; v, q)=\int_{\Omega} & \left(\frac{u^{k+1}-u^{k}}{\delta t}\right) \cdot v^{k+1}+\nu \nabla u^{k+\theta}: \nabla v^{k+1} \mathrm{~d} x \\
& +\int_{\Omega}\left(u^{k+\theta} \cdot \nabla\right) u^{k+\theta} \cdot v^{k+1} \mathrm{~d} x \\
& +\int_{\Omega} q^{k+1} \nabla \cdot u^{k+1}+p^{k+1} \nabla \cdot v^{k+1} \mathrm{~d} x,
\end{aligned}
$$

for all $(v, q)=\left\{\left(v_{k}, q_{k}\right)\right\}_{k=1}^{N} \in Y^{*}$, for $k=0, \ldots, N-1$. Note that that this part only involves the initial data $u_{0}$ from $m$. The operator $\mathcal{F}_{k, \Gamma_{D}}: Y \times M \rightarrow Y^{*}$ combines all the boundary integrals in 
(14) and reads

$$
\begin{aligned}
\mathcal{F}_{k, \Gamma_{D}}(m, y ; v, q)=- & \int_{\Gamma_{D}}\left(\nu \frac{\partial u^{k+\theta}}{\partial n}-p^{k+1} n\right) \cdot v^{k+1} \mathrm{~d} s \\
& -\int_{\Gamma_{D}}\left(\theta \nu \frac{\partial v^{k+1}}{\partial n}-q^{k+1} n\right) \cdot\left(u^{k+1}-g^{k+1}\right) \mathrm{d} s \\
& +\int_{\Gamma_{D}} \frac{\nu \sigma}{h}\left(u^{k+1}-g^{k+1}\right) \cdot v^{k+1} \mathrm{~d} s .
\end{aligned}
$$

The first integral in (17) arises when the integration by parts formula is applied to (13), and the integral would vanish if the Dirichlet boundary condition (2b) were strongly imposed on the space $V_{h}$. The remaining terms are added to obtain a variational problem that is consistent and stable, see e.g. [54, 55]. The form (17) is linear and symmetric, and positive definite provided that the parameter $\sigma$ is sufficiently large. We must also require $\theta>0$ to apply the Nitsche method.

The numerical examples in section 4 use two common finite element pairs: P2-P1 (Taylor-Hood) and P1-P1. The lowest order discretisation does not satisfy the LBB conditions, and hence requires stabilisation. We used the stabilisation $-\beta h^{2}(\nabla p, \nabla q)_{\Omega}$ where $h$ is the local mesh element size and $\beta=10^{-3}$ is the stabilisation coefficient.

3.3.2. Adjoint equations The adjoint approach is used to efficiently compute the functional derivative $D \hat{J}(m) \in M^{*}$, at the approximate cost of one linearised Navier-Stokes solve, namely solving the adjoint Navier-Stokes equations.

To derive the adjoint equations, consider the Navier-Stokes equations in the operator form $\mathcal{F}(m ; y)=0 \in Y^{*}$ and a functional $J(y, m) \in \mathbb{R}$. For brevity, we will use the simplified notation $F$ instead of $F(m ; y)$ in the following derivation.

The total derivative of the functional in direction $\tilde{m}$ is

$$
\langle D \hat{J}, \tilde{m}\rangle_{M^{*}, M}=\left\langle\frac{\mathrm{d} J}{\mathrm{~d} m}, \tilde{m}\right\rangle_{M^{*}, M}=\left\langle\frac{\partial J}{\partial y}, \frac{\mathrm{d} y}{\mathrm{~d} m} \tilde{m}\right\rangle_{Y^{*}, Y}+\left\langle\frac{\partial J}{\partial m}, \tilde{m}\right\rangle_{M^{*}, M} .
$$

Evaluating the right hand side of (18) directly is challenging because computing $\mathrm{d} y / \mathrm{d} m \in \mathcal{L}(M, Y)$ is computationally expensive. The adjoint approach eliminates this term by taking the derivative of the PDE equation

$$
\frac{\partial \mathcal{F}}{\partial y} \frac{\mathrm{d} y}{\mathrm{~d} m}+\frac{\partial \mathcal{F}}{\partial m}=0
$$

and substituting it into (18):

$$
\left\langle\frac{\mathrm{d} J}{\mathrm{~d} m}, \tilde{m}\right\rangle_{M^{*}, M}=-\left\langle\frac{\partial \mathcal{F}}{\partial m} \tilde{m},\left(\left(\frac{\partial \mathcal{F}}{\partial y}\right)^{-1}\right)^{*} \frac{\partial J}{\partial y}\right\rangle_{Y^{*}, Y}+\left\langle\frac{\partial J}{\partial m}, \tilde{m}\right\rangle_{M^{*}, M} .
$$

The functional derivative is then computed in two steps:

1. Compute the adjoint solution $\lambda \in Y$ by solving the adjoint PDE

$$
\left(\frac{\partial \mathcal{F}}{\partial y}\right)^{*} \lambda=-\frac{\partial J}{\partial y}
$$

2. Evaluate the derivative with

$$
\frac{\mathrm{d} J}{\mathrm{~d} m}=\left(\frac{\partial \mathcal{F}}{\partial m}\right)^{*} \lambda+\frac{\partial J}{\partial m}
$$

The computationally expensive part is the solution of (21), which involves the solution of a linear PDE.

The adjoint equations (21) can be derived before or after the discretisation of the Navier-Stokes equations [56]. Here, we apply the discretise-then-optimise approach, which has the advantage that 
the discretised derivative is the exact derivative of the discretised system. Alternative approaches do not guarantee this property and simple descent methods like algorithm 1 may fail and must be replaced by a more robust optimisation algorithm.

Applying (21) to the discretised Navier-Stokes operator (15) yields

$$
\left\langle\left(\frac{\partial \mathcal{F}}{\partial y}\right)^{*} \lambda, w\right\rangle=\left\langle\left(\frac{\partial \mathcal{F}}{\partial y}\right) w, \lambda\right\rangle=-\left\langle\frac{\partial J}{\partial u}, v\right\rangle \quad \forall w=(v, q) \in Y .
$$

Note that functional's regularisation term does not depend on $y$ and hence vanishes in the derivative. Since the adjoint operator is linear, $(\partial F / \partial y)^{*}$ can be written in matrix form, with each row corresponding to one timestep:

$$
\left(\frac{\partial \mathcal{F}}{\partial y}\right)^{*}=\left(\begin{array}{cccc}
\frac{\partial \mathcal{F}_{0}}{\partial y^{1}} & 0 & 0 & \cdots \\
\frac{\partial \mathcal{F}_{1}}{\partial y^{1}} & \frac{\partial \mathcal{F}_{1}}{\partial y^{2}} & 0 & \ddots \\
\vdots & \ddots & \ddots & \ddots \\
\cdots & 0 & \frac{\partial \mathcal{F}_{N-1}}{\partial y^{N-1}} & \frac{\partial \mathcal{F}_{N-1}}{\partial y^{N}}
\end{array}\right)^{*}=\left(\begin{array}{cccc}
\frac{\partial \mathcal{F}_{0}^{*}}{\partial y^{1}} & \frac{\partial \mathcal{F}_{1}^{*}}{\partial y^{1}} & 0 & \cdots \\
0 & \frac{\partial \mathcal{F}_{1}^{*}}{\partial y^{2}} & \ddots & \ddots \\
\vdots & \ddots & \ddots & \frac{\partial \mathcal{F}_{N-1}^{*}}{\partial y^{N-1}} \\
\cdots & 0 & 0 & \frac{\partial \mathcal{F}_{N-1}^{*}}{\partial y^{N}}
\end{array}\right)
$$

or more compactly,

$$
\left(\frac{\partial \mathcal{F}}{\partial y^{k}}\right)^{*} \lambda= \begin{cases}\left(\frac{\partial \mathcal{F}_{k-1}}{\partial y^{k}}\right)^{*} \lambda^{k}+\left(\frac{\partial \mathcal{F}_{k}}{\partial y^{k}}\right)^{*} \lambda^{k+1} & \text { for } 1 \leq k<N \\ \left(\frac{\partial \mathcal{F}_{k-1}}{\partial y^{k}}\right)^{*} \lambda^{k} & \text { for } k=N .\end{cases}
$$

Note that the last adjoint operator, for $k=N$, has the same structure as the other adjoint operators if one introduces a zero terminal adjoint value $\lambda^{N+1}$. Since the adjoint operator (24) is uppertriangular, the adjoint system (23) is solved by backwards substitution. Written explicitly, the adjoint equation is: find adjoint solutions $\lambda^{k}=\left(\lambda_{u}^{k}, \lambda_{p}^{k}\right)$ for $k=1, \ldots, N$ such that

$$
\begin{aligned}
& \int_{\Omega}\left(\frac{\lambda_{u}^{k}-\lambda_{u}^{k+1}}{\delta t}\right) \cdot v^{k} \mathrm{~d} x+\int_{\Omega} \nu \nabla \lambda_{u}^{k+\tilde{\theta}}: \nabla v^{k} \mathrm{~d} x \\
+ & \theta \int_{\Omega}\left(u^{k-1+\theta} \cdot \nabla\right) v^{k} \cdot \lambda_{u}^{k} \mathrm{~d} x+\theta \int_{\Omega}\left(v^{k} \cdot \nabla\right) u^{k-1+\theta} \cdot \lambda_{u}^{k} \mathrm{~d} x \\
+ & \tilde{\theta} \int_{\Omega}\left(u^{k+\theta} \cdot \nabla\right) v^{k} \cdot \lambda_{u}^{k+1} \mathrm{~d} x+\tilde{\theta} \int_{\Omega}\left(v^{k} \cdot \nabla\right) u^{k+\theta} \cdot \lambda_{u}^{k+1} \mathrm{~d} x \\
+ & \int_{\Omega} \lambda_{p}^{k} \nabla \cdot v^{k}+q^{k} \nabla \cdot \lambda_{u}^{k} \mathrm{~d} x-\int_{\Gamma_{D}}\left(\nu \frac{\partial v^{k}}{\partial n}\right) \cdot \lambda_{u}^{k+\tilde{\theta}} \mathrm{d} s \\
+ & \int_{\Gamma_{D}}\left(q^{k} n\right) \cdot \lambda_{u}^{k} \mathrm{~d} s-\int_{\Gamma_{D}}\left(\theta \nu \frac{\partial \lambda_{u}^{k}}{\partial n}-\lambda_{p}^{k} n\right) \cdot v^{k} \mathrm{~d} s \\
+ & \int_{\Gamma_{D}} \frac{\nu \sigma}{h} v^{k} \cdot \lambda_{u}^{k} \mathrm{~d} s=-\left\langle\frac{\partial J}{\partial u^{k}}, v^{k}\right\rangle \quad \forall v^{k}, q^{k} .
\end{aligned}
$$

with $\tilde{\theta}=1-\theta$ and $\lambda_{u}^{N+1}=0$. From (25), we observe that the adjoint system employs the same $\theta$ timestepping scheme as the forward discretisation, but with a modified advective velocity. Further, the adjoint system enforces homogeneous velocity Dirichlet boundary conditions on the controlled surfaces using a Nitsche like approach.

\subsection{Implementation and verification}

The Navier-Stokes solver was implemented in the FEniCS finite element framework [57] and the adjoint solver was automatically derived via the algorithmic differentiation tool dolfin-adjoint [58].

To verify that the gradients are computed correctly, we performed a Taylor test for 2D setup described in section 4.1. Table I lists the results of the Taylor test applied to the initial condition 


\begin{tabular}{l|cccc}
\hline$\left|\delta u_{0}\right|$ & $R_{0}\left(\delta u_{0}\right)$ & order & $R_{1}\left(\delta u_{0}\right)$ & order \\
\hline 0.005 & 0.5072 & & 0.00240 & \\
0.01 & 0.253 & 1.00 & 0.000599 & 2.00 \\
0.005 & 0.126 & 1.00 & 0.000150 & 1.99 \\
0.0025 & 0.0632 & 1.00 & $3.78 \mathrm{e}-05$ & 1.99 \\
0.00125 & 0.0316 & 1.00 & $9.00 \mathrm{e}-06$ & 2.01 \\
\hline
\end{tabular}

Table I. Taylor reminders $R_{0}=\left|\hat{J}\left(u_{0}+\delta u_{0}\right)-J\left(u_{0}\right)\right|$ and $R_{1}=\left|\hat{J}\left(u_{0}+\delta u_{0}\right)-\hat{J}\left(u_{0}\right)-D \hat{J}\left(u_{0}\right) \delta u_{0}\right|$ for the $2 \mathrm{D}$ example described in section 4.1 and a random initial condition perturbation $\delta u_{0}$. The instantaneous observation operators were used in the functional (5). The other setup parameters are listed in table II.

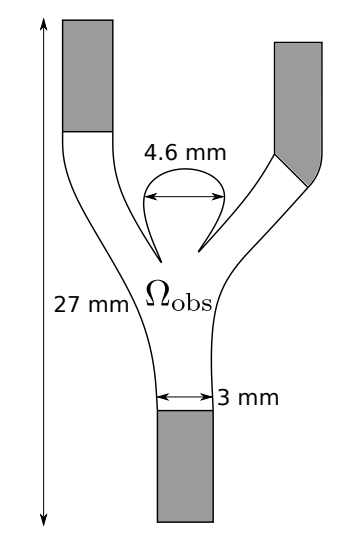

Figure 3. The computational domain for the 2D aneurysm experiment in section 4.1. The grey area indicates the extended domain $\Omega$ which is used to generate the physical observations.

control. The convergence rates are the expected orders of convergence for a correctly computed gradient.

The implementation and data to reproduce the Taylor test and the 2D results in section 4.1 are available online $e^{\dagger}$. The website contains a Readme file with instructions how to reproduce the results. The 3D data of section 4.2 could not be shared due to license restrictions.

\section{NUMERICAL EXPERIMENTS}

The data assimilation approach was tested on two numerical experiments. The first experiment consists of an idealistic, aneurysm-like 2D domain where the true velocity is known (section 4.1). The second experiment reconstructs the flow conditions in a real aneurysm from a 4D MRI scan (section 4.2).

\subsection{D Aneurysm}

The computational domain for this experiment resembles a 2D blood vessel bifurcation with an aneurysm, see figure 3 . The main goal of this experiment is to test the robustness of the assimilated solution against i) incomplete observations, ii) noisy observations, and iii) the regularisation parameters. In addition, we compare the quality of the reconstruction for two types of observation operators.

In order to be able to quantify the quality of the assimilated solution, we create a test environment where the "true" blood flow is known. This is achieved by generating the observations $d_{n}, n=$

†https://bitbucket.org/biocomp/navier_stokes_data_assimilation 


\begin{tabular}{lcrl} 
Parameter & Symbol & Value & Unit \\
\hline Viscosity & $\nu$ & 3.5 & $\mathrm{~m}^{2} / \mathrm{s}$ \\
Model timestep & $\Delta t$ & 0.00925 & $\mathrm{~s}$ \\
End time & $T$ & 0.629 & $\mathrm{~s}$ \\
Time discretisation & $\theta$ & 0.5 & \\
Spatial discretisation & & $P 1-P 1$ & \\
Mesh triangles in $\Omega$ & & 20,989 & \\
Mesh triangles in $\Omega_{\mathrm{obs}}$ & & 13,296 & \\
Mesh element size & & $0.03-0.20$ & $\mathrm{~mm}$ \\
Nitsche coefficient & $\sigma$ & 100.0 & \\
\hline Number of physical observations & $\mathrm{N}$ & 17 & \\
Regularisation & $\alpha=\gamma$ & $10^{-5}$ & \\
\hline
\end{tabular}

Table II. The model settings for the 2D aneurysm experiment in section 4.1 if not otherwise stated.

$1, \ldots, N$ from a numerical reference solution. The reference solution was obtained by solving the Navier-Stokes equations on an extended domain that included the grey area in figure 3 . On the inlet and right outlet boundaries, a parabolic velocity profiles was enforced with peak values of $1000 \mathrm{~mm} / \mathrm{s}$ and $870 \mathrm{~mm} / \mathrm{s}$, respectively, multiplied by $\sin \left(\pi(1-t)^{3}\right)$ to obtain a pulse like flow pattern ${ }^{\ddagger}$. The simulation started at $t=0 \mathrm{~s}$ with zero velocity, reached the peak inflow velocity at $t=0.206 \mathrm{~s}$, and stopped at $t=0.629 \mathrm{~s}$ when the inflow velocity dropped below $5 \%$ of the peak velocity. The remaining model settings are listed in table II. The value for the regularisation coefficients $\alpha=\gamma$ were determined manually such that the reconstruction is robust while ensuring that the regularisation does not dominate the solution. The effect of this choice will be studied in more detail in section 4.1.1. Alternative approaches for choosing $\alpha$ and $\gamma$ are the discrepancy principle $[59,60,61]$, which selects the regularisation parameter such that the perturbation of the regularisation term affects the solution with the same order of the discrepancy induced by the noise, or the computationally more efficient approach proposed by [27, 26].

The physical observations were obtained by applying the observation operators $\mathcal{T}_{n}$ to the reference solution. We compared two types of observation operators: first, the instantaneous observation operators $\mathcal{T}_{n}^{\text {inst }}$ which take instantaneous snapshots of the velocity profile in $\Omega_{\mathrm{obs}}$ at evenly distributed levels, that is:

$$
\mathcal{T}_{n}^{\text {inst }} u(x):=\mathcal{R}_{\Omega_{\mathrm{obs}}} u\left(x, t_{n}\right) \quad \forall x \text { in } \Omega_{\mathrm{obs}},
$$

with $t_{n}=T n / N, n=0, \ldots, N-1$. Here $\mathcal{R}_{\Omega_{\mathrm{obs}}}$ restricts the velocity to the observation domain, the white area in figure 3. Second, the time-averaging observation operators, which measure timeaveraged velocities over a time interval:

$$
\mathcal{T}_{n}^{\mathrm{avg}} u(x):=\frac{1}{t_{n}-t_{n-1}} \int_{t_{n-1}}^{t_{n}} \mathcal{R}_{\Omega_{\mathrm{obs}}} u(x, t) \mathrm{d} t \quad \forall x \text { in } \Omega_{\mathrm{obs}}
$$

with evenly distributed time levels $t_{n}=T n / N, n=0, \ldots, N$. Both observation operators simulate spatial and temporal incompleteness of physical measurements, due to the restriction to $\Omega_{\mathrm{obs}}$ and by limiting to $N$ observations which is less than the number of model time steps.

The variational data assimilation was then used to recover the reference solution. To avoid an "inverse crime", we performed the reconstruction on the restricted domain $\Omega_{\mathrm{obs}}$. This meant that the assimilation procedure did not have knowledge about full geometry used for the data generation. In addition, the outflow Dirichlet boundaries were swapped between the data generation and reconstruction. In order words, the traction-free outlet boundary differed during data generation and data assimilation.

${ }_{\ddagger}^{\ddagger}$ Note that real flow in cerebral arteries will never go to zero, but rather pulsate between around $0.5 \mathrm{~m} / \mathrm{s}$ and one third of $0.5 \mathrm{~m} / \mathrm{s}$. 
The discretised optimisation problem had 32,440 dimensions, describing the initial condition and the boundary conditions at each time-step. The non-linear problem arising from the discretised Navier-Stokes equation at each time-step had 13,784 degree of freedoms, which was solved with a Newton-LU solver. The optimisation started with the boundary and initial conditions set to zero, and was terminated when the relative change of the functional in one iteration dropped below $10^{-4}$ or the number of iterations exceeded 100.

The remainder of this section compares this "observation generation followed by data assimilation" procedure for different setups. To simplify the comparisons, all results are presented in an consistent way. As an example, consider figure 4 . Each column in this figure summarises one data assimilation output and lists key characteristics: Opt.-iters denotes the number of iterations for the optimisation solver to converge. The $J+R$ value is the functional value (5) and the regularisation term (7) at the optimal point. The next two graphics (Observation and Assimilation) visualise the observed and reconstructed flow speeds at $t=0.296 \mathrm{~s}$, which corresponds to the observation time with peak velocities inside the aneurysm. The colour scale is logarithmic in order to better highlight differences in the results. Note that the reconstruction can be accurate even if these two graphics differ, for instance when noise was added to the physical observations. The last two time-plots compare assimilated velocity profile with the "true" velocity from the reference simulation. Since the results in the aneurysm is typically most important, these time-plots focus on the aneurysm area, denoted as $\Omega_{\text {ane }}$. The first time-plot shows the $L^{2}$-norms of the true velocity (from the observation generation step), the assimilated velocity and their difference over the simulation time. We also state the relative error over space and time:

$$
\mathcal{E}_{\Omega_{\text {ane }}}=\frac{\left\|u_{\text {true }}-u\right\|_{L^{2}\left(\Omega_{\mathrm{ane}} \times(0, T]\right)}}{\left\|u_{\text {true }}\right\|_{L^{2}\left(\Omega_{\text {ane }} \times(0, T]\right)}}
$$

where $u_{\text {true }}$ is the true velocity. The wall shear stress on the aneurysm wall is an important diagnostic value for aneurysm rupture risk studies. It is defined as $\operatorname{WSS}(u)=|\sigma n-(\sigma n \cdot n) n|$ with $\sigma=\rho\left(-p I+\nu\left(\nabla u+(\nabla u)^{T}\right)\right)$ and $\rho=1060 \mathrm{~kg} / \mathrm{m}^{3}$. The final time-plot of each figure column shows the $L^{2}$-norm of the wall shear stress over the simulation time. As before, we defined a measure of the relative space-time error of the reconstructed wall shear stress on the aneurysm wall

$$
\mathcal{E}_{\mathrm{WSS}}=\frac{\left\|\mathrm{WSS}\left(u_{\text {true }}\right)-\mathrm{WSS}(u)\right\|_{L^{2}(\text { (ane } \times(0, T])}}{\left\|\mathrm{WSS}\left(u_{\text {true }}\right)\right\|_{L^{2}(\text { Гane } \times(0, T])}},
$$

where $\Gamma_{\text {ane }}$ denotes the aneurysm wall.

The assimilation results with instantaneous observation operators $\mathcal{T}_{n}^{\text {inst }}$ and the parameters listed in II are shown in figure 4 (left column, Base setup with no noise). The optimisation converged in 46 iterations, which includes in total 52 forward and adjoint Navier-Stokes solves. The optimisation reduced the initial functional value of $9.3 \times 10^{7}$ to $7.4 \times 10^{5}$. Of the latter only 495 is due to the regularisation term $\mathcal{R}$ and the rest originates from the goal functional $\mathcal{J}$, indicating that the assimilation is not-dominated by the regularisation term. The two top plots in figure 4 (left column) show that the assimilation procedure recovered the observation well, only minor differences in the aneurysm and the outflow boundaries are visible. Also the timeplots show that the assimilated velocities/WSS matches well with the "true" velocity/WSS also in between the observation snapshots. Both error metrics indicate a relative error of $10 \%$ or less.

The same plots, but with the assimilation performed based on the time-averaging observation operators $\mathcal{T}_{n}^{\text {avg }}$, are shown in 5 (left column, Base setup with no noise). Overall, the quality of the reconstruction is comparable as with $\mathcal{T}_{n}^{\text {inst }}$ but with a slight decrease in number of optimisation iterations and slightly higher relative error in the reconstructed wall shear stress.

4.1.1. Assimilation robustness The quality of the reconstruction might be influenced by different factors, such as noise in the physical observations, the choice of the controlled boundaries, or the amount of regularisation. To study the robustness of the assimilation procedure, we varied a set of parameters independently, repeated the data assimilation and compared the "true" solution from the 
reference simulation. More specifically, the following tests are based on the configuration listed in table II, and in each test one parameter is varied and the quality of the reconstruction investigated.

Noisy observations (figures 4 and 5). Pointwise Gaussian white noise was added to the physical observations with zero mean and different levels of intensity. While this type of noise is not expected in real physical measurements, in particular because it depends on the numerical mesh, we consider it as a suitable benchmark setup. The signal-to-noise ratio was computed as the ratio of the energy of the unperturbed measurement data to the energy of the added Gaussian noise. The results of the reconstruction for different signal-to-noise ratios are shown in figure 4 for the instantaneous observation operators $\mathcal{T}_{n}^{\text {inst }}$, and figure 5 for the time-averaging observation operators $\mathcal{T}_{n}^{\text {avg }}$. With higher noise levels, the functional value $\mathcal{J}+\mathcal{R}$ increases due to the larger difference between reconstructed and observed velocity. Nevertheless, the error measures $\mathcal{E}_{\text {ane }}$ and $\mathcal{E}_{\text {WSS }}$ remain small, indicating that the assimilation is robust against this type of noise, even for high noise to signal ratios. One reason for this robustness is that the main determinant for the flow is the flow division between the two outlets, which remains nearly unchanged when adding uniform pointwise Gaussian noise. Furthermore, the data assimilation acts like a denoising procedure thanks to the mathematical model. This de-noising effect has also been observed by [26].

Regularisation (figures 6 and 7). The regularisation terms (7) enforce "smoothness" on the control functions. As such, the regularisation coefficients $\alpha$ and $\gamma$ could have a strong influence on the assimilation results. This was investigated by varying $\alpha$ and $\gamma$ between 1 and $10^{-5}$, simultaneously in order to keep a balance between the two terms. The results are shown in figures 6 for $\mathcal{T}_{n}^{\text {inst }}$ and 7 for $\mathcal{T}_{n}^{\text {avg }}$. The reconstruction works well for $\alpha=\gamma=10^{-4}$ and $10^{-5}$ (latter is shown in "Base setup with no noise" in figures 4 and 5). The quality starts to reduce for $\alpha=\gamma=10^{-2}$, in particular the relative errors $\mathcal{E}_{\text {ane }}$ and $\mathcal{E}_{\mathrm{WSS}}$ are approximately twice as high as compared to $\alpha=\gamma=10^{-4}$. For $\alpha=\gamma=1$ the error metrics increase further and both time-plots show a visibly lower assimilated velocity and WSS compared to the reference values.

Data sparsity (figures 8 and 9). Another important question is how many physical observations $N$ are required to accurately reconstruct the blood flow. To address this question, the data assimilation was repeated with varying number of observations. The base setup (left column in figures 4 and 5) used $N=16$ and the results for $N=4,8$ and 32 are shown in figures 8 and 9 for $\mathcal{T}_{n}^{\text {inst }}$ and $\mathcal{T}_{n}^{\text {avg }}$, respectively.

With $N=4$, the quality of the reconstruction suffers visibly with relative velocity errors of $26 \%$ for the instantaneous and $16 \%$ for the averaged observation operators. With $N=8$, the results of the averaged observation operator improves significantly and the relative velocity error is with $11 \%$ nearly as good as the base case. In contrast, the results with the instantaneous do not show this improvement. Finally, the differences between $N=16$ to $N=32$ observations are minimal for both observation operators. Overall, $N=16$ observations seems a good choice for this setup, and the averaged observation operator seems more robust against lower number of observations.

Choice of controlled outflow boundary (figure 10). In the problem definition (2b), an arbitrary choice was made to control the outflow on $\Gamma_{\text {out }_{1}}$, and to enforce a no-stress condition on $\Gamma_{\text {out }_{2}}$. It is therefore natural to verify that the reconstruction succeeds also if $\Gamma_{\text {out }_{2}}$ is controlled and a non-stress condition is applied on $\Gamma_{\text {out }_{1}}$. The results for this setup are shown in figure 10. The reconstruction is similarly good as in the base setup, indicating that the assimilation is not impacted significantly by the choice of the controlled boundaries. Nevertheless, this choice might be more significant for other setups, in particular if one of the outflows is in close proximity to the aneurysm.

\subsection{Flow reconstruction in an aneurysm from $4 D M R A$ measurements}

The variational data assimilation approach was tested on real measurements of a dog's blood vessel with an artificially introduced aneurysm. The physical measurements were obtained using 4D PCMRI and resulted voxel-based velocity snapshots covering the complete computational domain with a $(0.6 \mathrm{~mm})^{3}$ resolution. The pulsatile flow was imaged at 16 equidistant time-levels during the cardiac cycle between $t=0 \mathrm{~s}$ and $0.629 \mathrm{~s}$. A complete description of the imaging technique and corresponding 4D PC-MRI data used here can be found in [15]. We used both instantaneous 
Base setup without noise

$$
\begin{gathered}
\text { Opt.-iters.: } 46 \\
J+\mathcal{R}=7 \times 10^{5}
\end{gathered}
$$

$$
\begin{gathered}
\text { Base setup with } \\
\text { signal-to-noise ratio of } 2
\end{gathered}
$$

$$
\begin{gathered}
\text { Opt.-iters.: } 50 \\
J+\mathcal{R}=445 \times 10^{5}
\end{gathered}
$$

$$
\begin{gathered}
\text { Base setup with } \\
\text { signal-to-noise ratio of } 1
\end{gathered}
$$

Opt.-iters.: 27

$J+\mathcal{R}=887 \times 10^{5}$
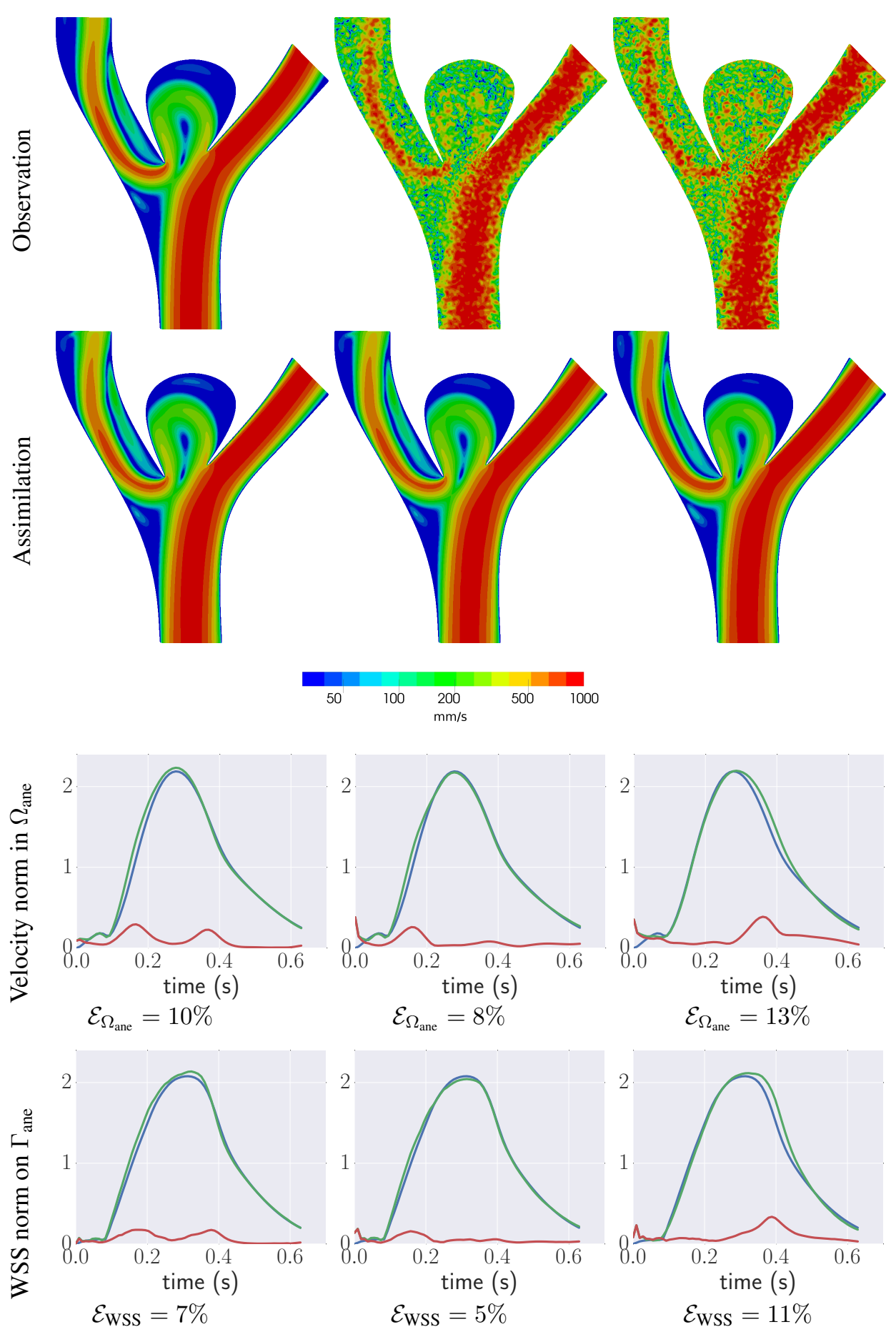

True

Assimilated

Error

Figure 4. Results using the instantaneous observation operators with pointwise additive Gaussian white noise. The signal-to-noise-ratio was computed as $\left\|\mathcal{T}_{n}^{\text {inst }} u_{\text {true }}\right\|^{2} /\left\|\mathcal{T}_{n}^{\text {inst }} u_{\text {true }}-d\right\|^{2}$, where $d$ is the noisy data. The snapshots on the top two rows are taken at $t=0.296 \mathrm{~s}$. 

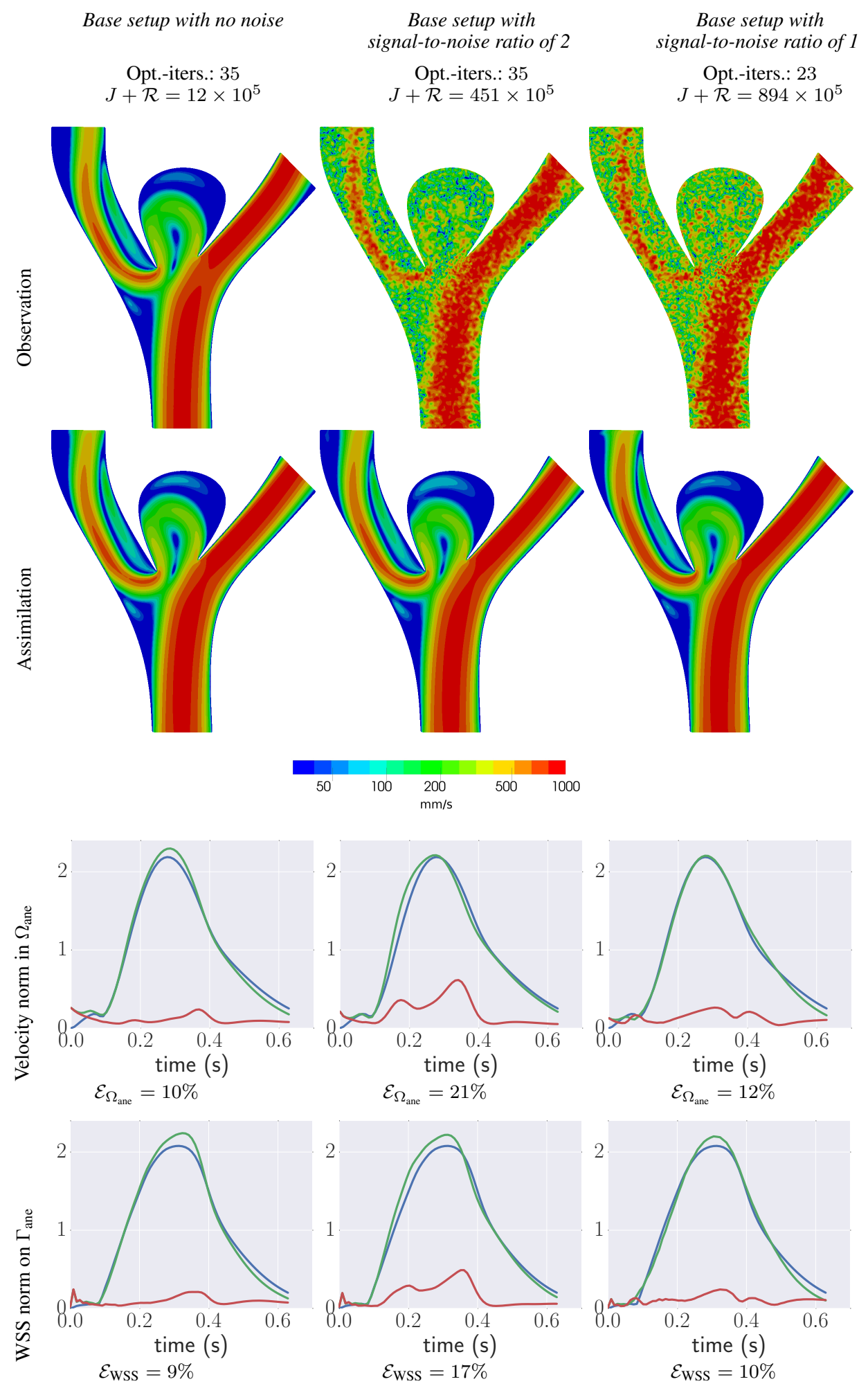

True

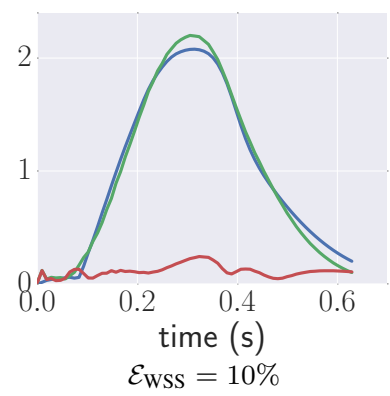

Error

Figure 5. Results using the time-averaging observation operators with pointwise additive Gaussian white noise. The signal-to-noise ratio was computed as $\left\|\mathcal{T}_{n}^{\text {avg }} u_{\text {true }}\right\|^{2} /\left\|\mathcal{T}_{n}^{\text {avg }} u_{\text {true }}-d\right\|^{2}$, where $d$ is the noisy data. The snapshots on the top two rows are taken at $t=0.296 \mathrm{~s}$. 

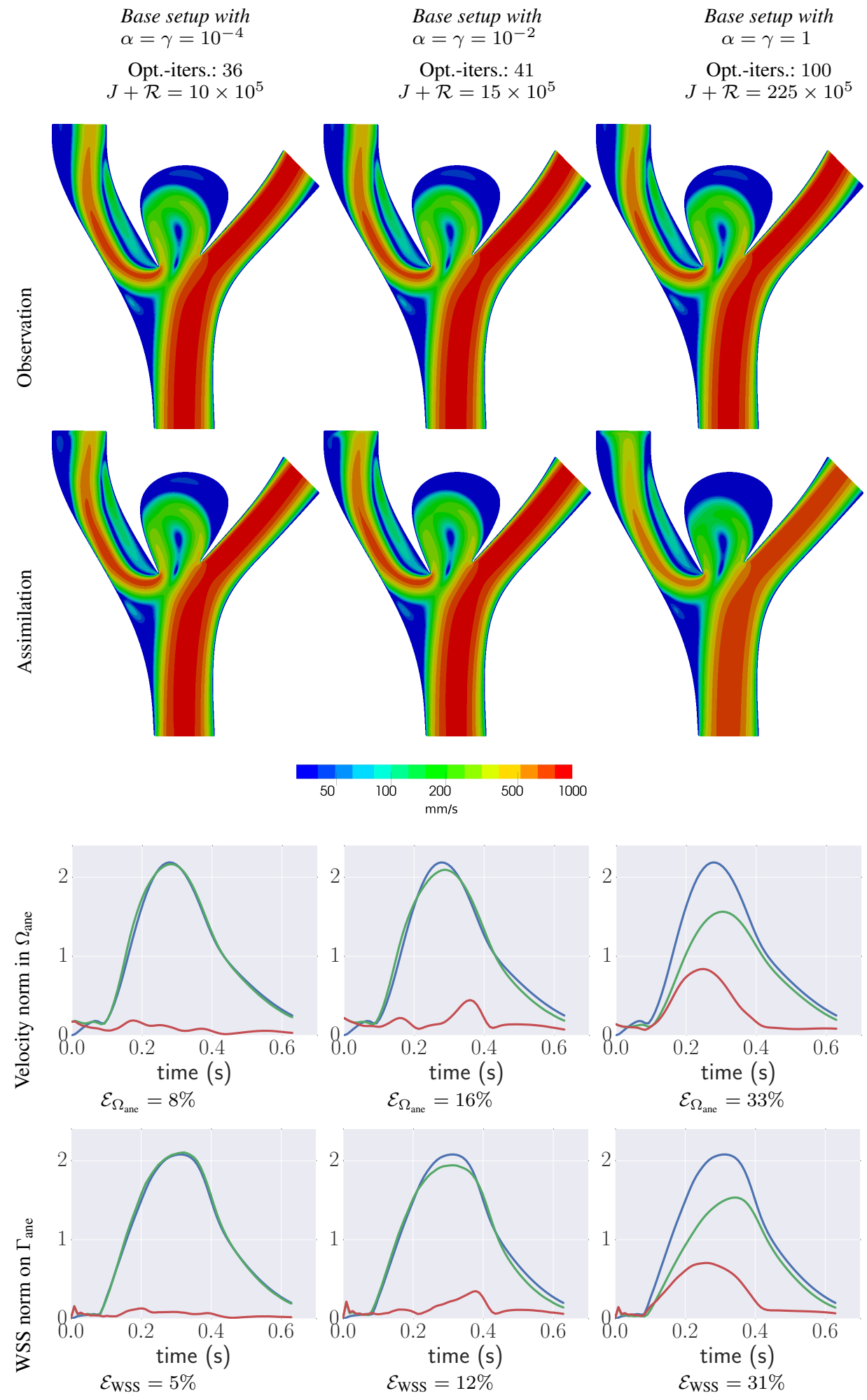

True

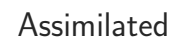

Error

Figure 6. Results using the instantaneous observation operators with varying $\alpha$ and $\gamma$ regularisation coefficients. The base setup (figure 4, left column) uses $\alpha=\gamma=10^{-5}$. The snapshots on the top two rows are taken at $t=0.296 \mathrm{~s}$. 

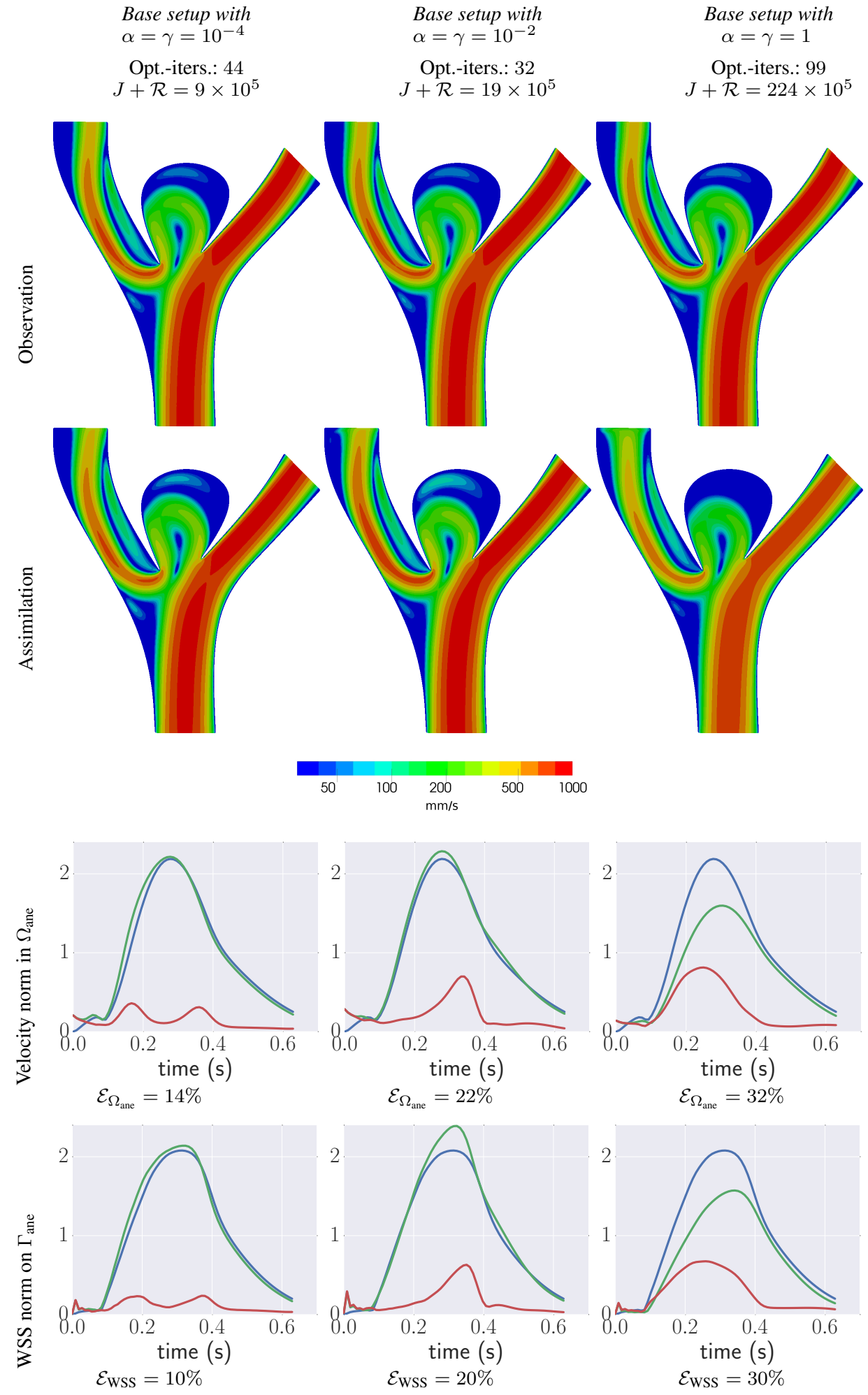

$\longrightarrow$ True

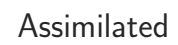

Error

Figure 7. Results using the time-averaging observation operators with varying $\alpha$ regularisation parameters. The base setup (figure 5, left column) uses $\alpha=\gamma=10^{-5}$. The snapshots on the top two rows are taken at $t=0.296 \mathrm{~s}$. 

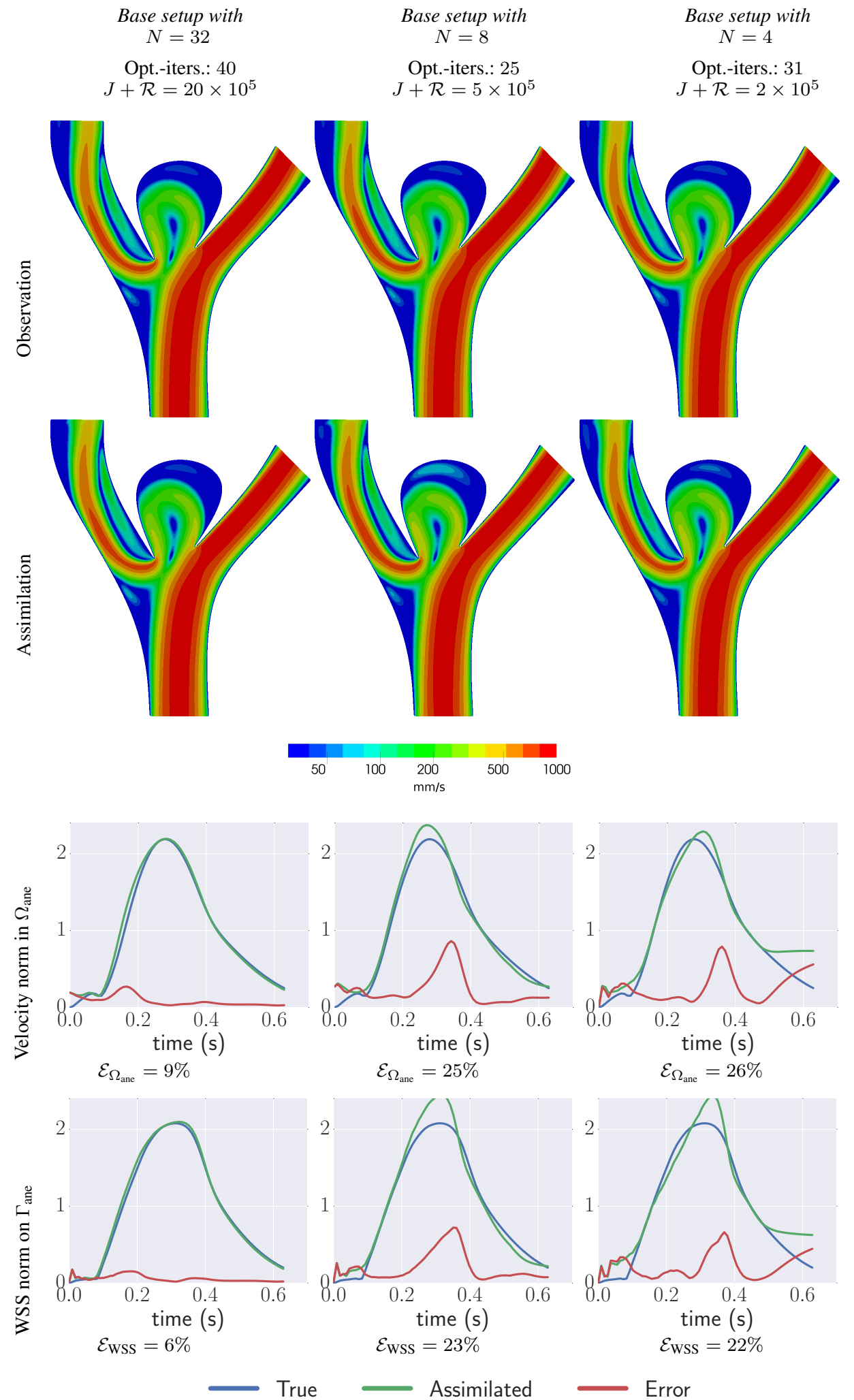

Error

Figure 8. Results using the instantaneous observation operators with varying number of observations. The base setup (figure 4 , left column) uses $N=16$ observations. The snapshots on the top two rows are taken at $t=0.296 \mathrm{~s}$. 

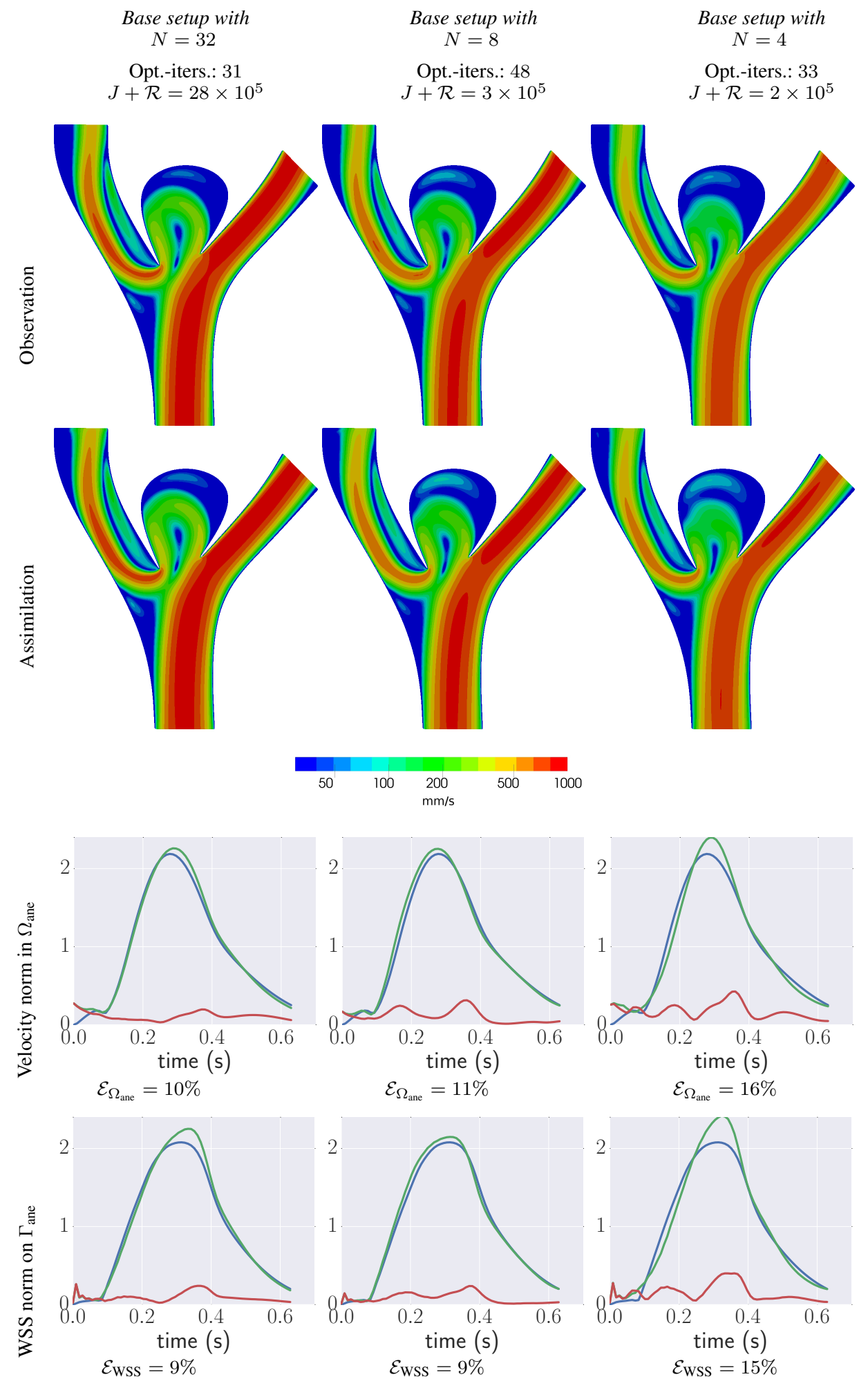

- True

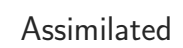

Error

Figure 9. Results using the time-averaging observation operators with varying number of observations. The base setup (figure 5, left column) uses $N=16$ observations. The snapshots on the top two rows are taken at $t=0.296 \mathrm{~s}$. 

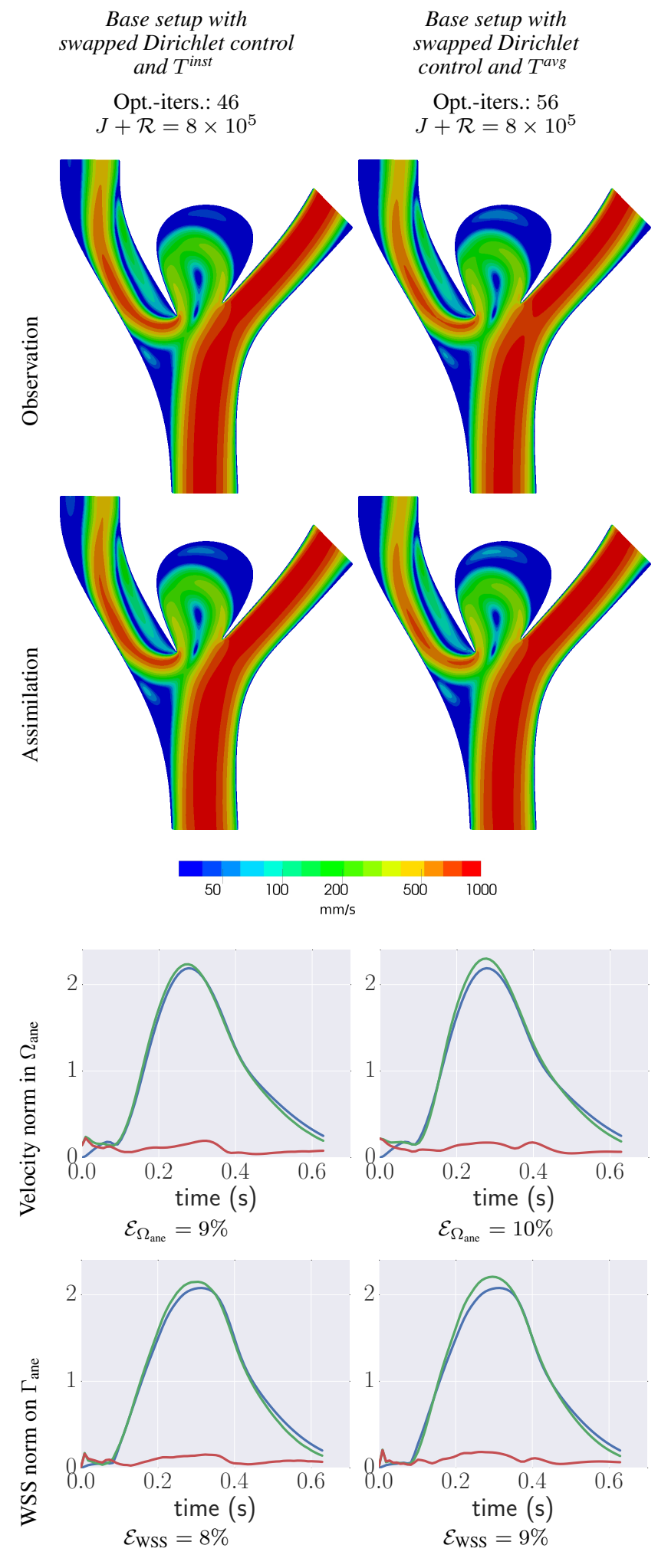

True

Assimilated

Difference

Figure 10. Assimilation results where the controlled outlets are swapped from the base cases (figures 4 and 5 , left columns). The snapshots on the top two rows are taken at $t=0.296 \mathrm{~s}$. 


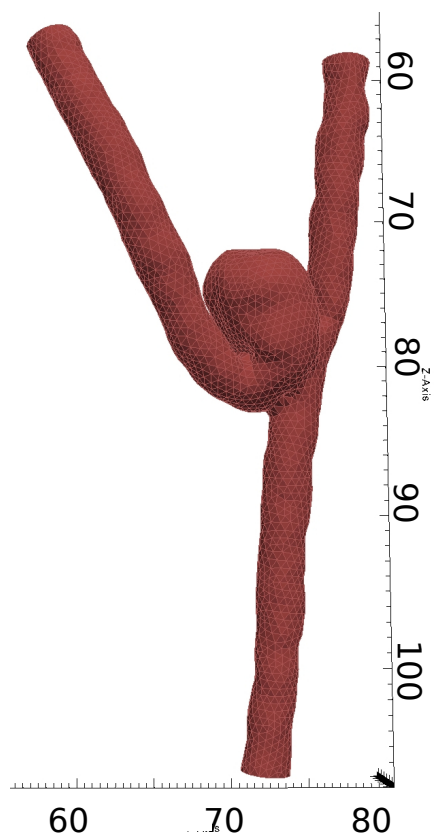

Figure 11. The segmented geometry with overlayed mesh for example 4.2.

\begin{tabular}{lccl} 
Parameter & Symbol & Value & Unit \\
\hline Viscosity & $\nu$ & 7.5 & $\mathrm{~m}^{2} / \mathrm{s}$ \\
Model timestep & $\Delta t$ & 0.004625 & $\mathrm{~s}$ \\
End time & $T$ & 0.629 & $\mathrm{~s}$ \\
Time discretisation & $\theta$ & 1.0 & \\
Spatial discretisation & & $P 1-P 1$ & \\
Number of mesh cells in $\Omega$ & 261,124 & & \\
Nitsche coefficient & $\sigma$ & 100.0 & \\
\hline Number of observations & $\mathrm{N}$ & 16 & \\
Regularisation parameter & $\alpha=\gamma$ & $10^{-5}$ &
\end{tabular}

Table III. The numerical settings for the reconstruction of blood flow from 4D MRA measurements. The first parameters specify the model setup, while the final two parameters configure the data assimilation.

snapshots and time-averaged measurements over each voxel and/or time-interval. Hence, both types of observation operators $\mathcal{T}_{n}^{\text {inst }}$ and $\mathcal{T}_{n}^{\text {avg }}$ are relevant for this experiment.

The computational domain $\Omega$ was segmented from the time-averaged MRA measurements using VMTK (wWw. vmtk.org) and is shown in figure 11. The physical observations $d_{n}$ were obtained by linearly interpolating each 4D PC-MRI snapshot onto the mesh nodes. As a consequence, the physical observations were available in the entire computational domain, that is $\Omega_{\mathrm{obs}}=\Omega$. The remaining numerical settings are listed in table III. The discretised optimisation problem had 208,536 dimensions, describing the initial condition and the boundary conditions at each time-step. The non-linear problem arising from the discretised Navier-Stokes equation at each time-step had 184,464 degree of freedoms, which was solved with a Newton-LU solver. The optimisation assimilation problem was solved on the Abel supercomputing facility on 24 CPUs and was terminated after 30 optimisation iterations, when the relative change in one optimisation iteration dropped below $0.09 \%$. The optimisation reduced the initial objective value of $538 \cdot 10^{6}$ to $192 \cdot 10^{6}$ of which $25.8 \cdot 10^{3}$ can be contributed to the regularisation terms.

For comparison, we additionally performed a "traditional", high-resolution and low-viscosity flow simulation without the data assimilation procedure, but instead with interpolated initial and boundary conditions from the observations. To avoid spurious effects near the boundary, the 
segmented geometry needed to be extended by artificial straight arteries on the in- and outlets. The high-resolution setup had 20 million DOFs with a Taylor-Hood pressure-corrector scheme and a timestep of $5.9 \cdot 10^{-4} \mathrm{~s}$. Womersley boundary conditions were used on the inflow and outflows. The inflow flux $Q_{\Gamma_{\text {in }}}$ was interpolated from averaged observations as $\left(Q_{\Gamma_{\text {in }}}-Q_{\Gamma_{\text {out }_{1}}}-Q_{\Gamma_{\text {out }_{2}}}\right) / 3$, the outflow flux on $\Gamma_{\text {out }_{1}}$ was averaged as $Q_{\Gamma_{\text {out }_{1}}} /\left(Q_{\Gamma_{\text {out }_{1}}}+Q_{\Gamma_{\text {out }_{2}}}\right)$, and a traction free condition was applied on $\Gamma_{\text {out }_{2}}$.

Figure 12 visualises the flow speeds obtained from the assimilated, and the high-resolution simulations for both observation operators. We observe that the differences between the instantaneous and averaged observation operators are marginal, and that all simulations recover the main flow characteristics. The remaining discrepancies between the observations and simulated flow speeds are partially caused by the noise in the observations, which is not present in the simulated flows. In addition, there are also more localised discrepancies, especially on the right aneurysm wall and the outflow vessel to the right, which are unlikely caused by noise. We suspect that these differences are the result of errors in the wall definition during the geometry segmentation, and simplified model assumptions (e.g. non-Newtonian rheology, choice of viscosity).

When comparing the high-resolution solution with the assimilated solution, we observe that the high-resolution solution has an extensive high-speed flow along the right aneurysm wall, which is neither present in the assimilated solution nor the observations. The high-resolution solution has also transient to turbulent behaviour in the aneurysm, while the assimilated solution is laminar. The observations are too coarse to determine if an turbulent or laminar solution is expected in this case. Overall, it is difficult to declare a winner between the high-resolution and assimilated solution.

\section{CONCLUSION, DISCUSSION AND FUTURE WORK}

This paper presented the application of variational data assimilation to reconstruct transient blood flow from velocity observations such as from MRI images. We formulated the assimilation problem as an optimisation problem to recover the initial and boundary conditions of the Navier-Stokes equations from a set of observations, and introduced appropriate regularisation terms. Numerically, we employed a mesh-independent BFGS optimisation algorithm and derived the adjoint NavierStokes equations to efficiently compute the required functional derivatives. Numerical experiments of a synthetic 3D (time and 2D space) case indicate that the technique is robust with respect to pointwise Gaussian measurement noise and to the regularization parameters. The results showed also that, as expected, the reconstruction becomes worse if the number of measurements is decreased. Another experiment applied variational data assimilation on the geometry and measurements that origin from a 4D PC-MRI dataset obtained from a canine aneurysm model. This experiment demonstrated that the methodology is also feasible for 4D (time and 3D space) problems.

We have to bear in mind that the resolution of the 4D PC-MRI is currently limited to $0.3-0.6 \mathrm{~mm}$, around 30 time steps per heart cycle and that the PC MRI flow represent phase-average of several hundred cardiac cycles , c.f. e.g. [16]. Potential transitional effects may therefore be masked by the averaging procedure involved in the MRI acquisition. It is however interesting to note that the data assimilation procedure is relatively robust with respect to noise as the main determinant for the flow is the flow division between the different outlets. Overall, the variational data assimilation technique seems to be a promising approach for incorporating measurements into transient blood flow models.

The methodologies in this paper are simplified and can benefit from further improvements. For example, the flow model assumed Newtonian fluid within rigid walls. This is a gross simplification, though it has been found adequate to capture the main flow structures in several studies [40, 15] and is used in most large studies involving many patients [1, 2, 3]. A possible extension is therefore to take into account the deformation of the vessel wall [30, ?] to determined patientspecific compliance parameters and to determine non-Newtonian effects [41] in terms of nonlinear rheology models. Furthermore, the geometry of the blood-vessel can be treated as an unknown variable and recovered from the observations, for example with the approach taken in [29] or via shape optimisation techniques [38]. Other improvements concern the variational setup itself. The 


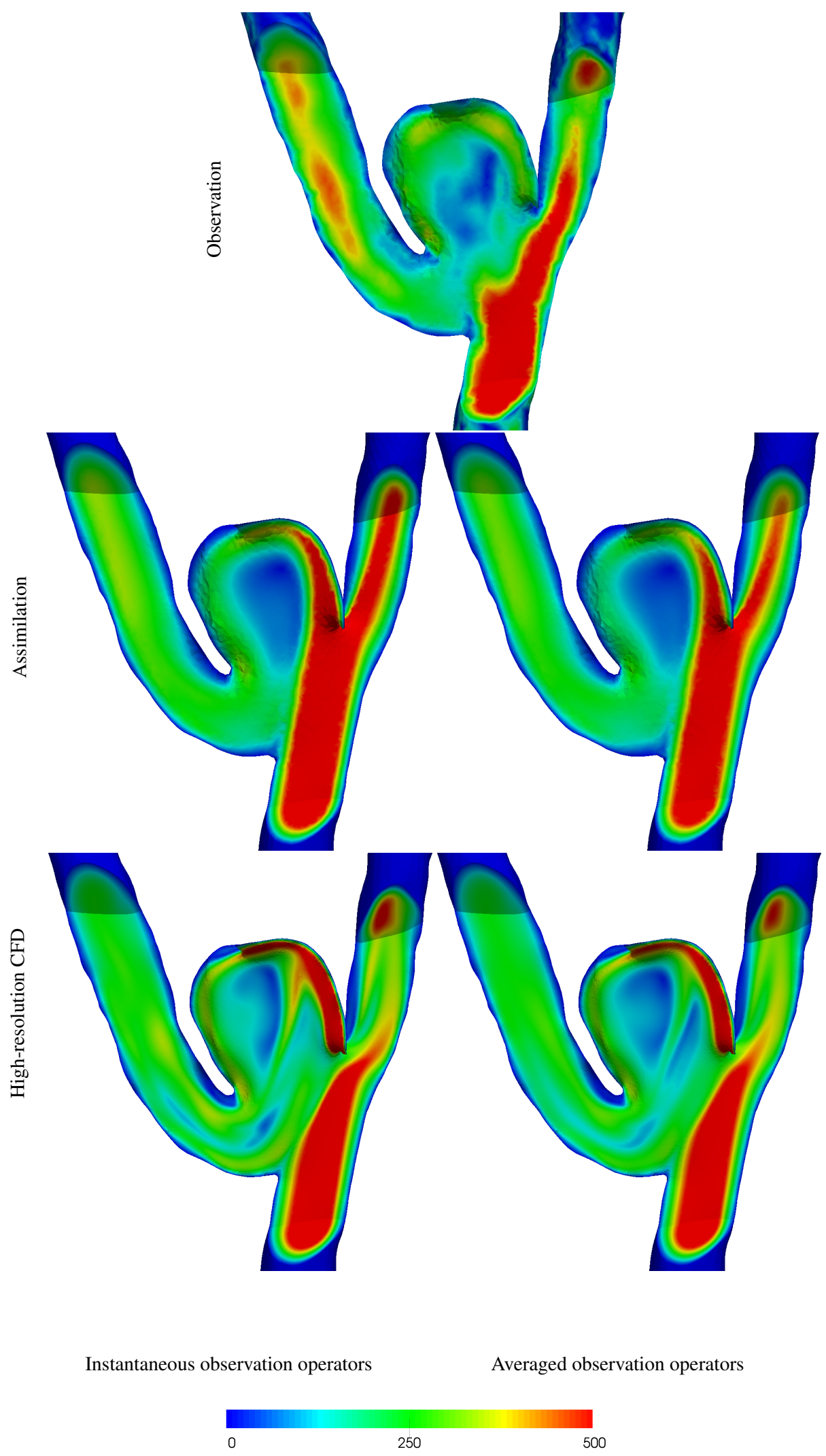

Figure 12. Flow speed visualised through a slice of the 3D dog vessel, see section 4.2. Shown are the observations at $t=0.296 \mathrm{~s}$. 
observations operators are idealised, and should be adapted to the specific measurement device used to obtain the observations. In addition, the initial condition should be replaced by periodic boundary conditions, but this option was disregarded in the current project because of currently lacking support in our software tools. The assumption of traction free boundary on $\Gamma_{\text {out }_{2}}$ could be removed by optimising the boundary conditions at all open boundaries. The latter requires however an additional consistency constraint to ensure mass balance on the boundary.

Several recent numerical studies have reported transitional flow in cerebral aneurysms $[18,19,16]$ and in this paper we have shown that there is a difference between the assimilated solution and a corresponding high-resolution simulation that demonstrated transient to turbulent behaviour. There are two main reasons that transitional or turbulent flow is not captured by our assimilation procedure: 1) the resolution in space and time is too coarse, and 2) transient and/or turbulent flow would likely require the simulation of many cardiac cycles, possibly combined with periodic boundary conditions. To what extent the methodology described here extends to such formulations and more efficient schemes like projection/pressure correction schemes is currently an open question.

Finally, the numerical schemes can be improved. We used a simple, low-order time stepping scheme, but in practice it might be preferable to employ higher order Runge-Kutta schemes or similar methods. The variational data assimilation approach can be extended to such methods, but the associated adjoint equations must be derived accordingly, see e.g. [50, 51, 52]. Adaptive time stepping schemes may also be employed, but require special attention when developing the adjoint equations $[62,63]$. The computational expense of a variational data assimilation with a more advanced numerical scheme is expected to scale with the cost of the discretisation scheme. The extension to higher-order finite elements is straightforward but is more computationally expensive and was therefore left out of this study.

The methodology proposed in this paper appears to be quite robust in the sense of numerical stability for both the transient $2 \mathrm{D}$ and $3 \mathrm{D}$ problems discussed. We remark that this was not the case in several earlier attempts where we used projection schemes for a faster solution of the flow problems, alternative regularizations and with other formulations than the reduced optimisation problem. Similar problems were not experienced in the stationary case.

The computational cost of the variational data assimilation for the given examples was typically between 50-100 times that of a single flow simulation. To keep the computational time feasible, the mesh and time resolutions had to be reduced compared to a single flow simulation study. In order to obtain spatial and temporal resolved discretisations, it is therefore necessary to increase the overall efficiency of the method. We chose to solve the assimilation problem in its reduced formulation, which has the advantage that existing Navier-Stokes solution methods can be directly reused in the assimilation, and that the associated adjoint solver could automatically be derived with the 'dolfin-adjoint' software. An alternative approach would have been to derive and solve the firstorder optimality system $[56,64]$. This leads to a large, non-linear system that couples all spatial and temporal degrees of freedom of the discretised Navier-Stokes and adjoint Navier-Stokes equations. Solving this system requires the development of specialised solvers, but can in some cases lead to superior performance compared to reduced approach (see for example [65]) and is therefore an interesting avenue for future improvements.

\section{ACKNOWLEDGMENTS}

This research was supported by the Research Council of Norway through a Centres of Excellence grant to the Center for Biomedical Computing at Simula Research Laboratory, project number 179578, and a FRIPRO grant, project number 251237. Computations were performed on the Abel supercomputing cluster at the University of Oslo via NOTUR projects NN9279K and NN9316K. In addition, the authors would like to thank Jingfeng Jiang and Charles Strother for providing the 4D phase-contrast MRA data, Lorenz John for comments on the regularisation and the reviewers for their constructive comments. 


\section{REFERENCES}

1. Cebral JR, Mut F, Weir J, Putman C. Quantitative characterization of the hemodynamic environment in ruptured and unruptured brain aneurysms. American Journal of Neuroradiology 2011; 32(1):145-151, doi:10.3174/ajnr.A2419.

2. Xiang J, Natarajan SK, Tremmel M, Ma D, Mocco J, Hopkins LN, Siddiqui AH, Levy EI, Meng H. Hemodynamic-morphologic discriminants for intracranial aneurysm rupture. Stroke 2011; 42(1):144-52, doi: 10.1161/STROKEAHA.110.592923.

3. Takao H, Murayama Y, Otsuka S, Qian Y, Mohamed A, Masuda S, Yamamoto M, Abe T. Hemodynamic differences between unruptured and ruptured intracranial aneurysms during observation. Stroke 2012; 43(5):1436-1439, doi: 10.1161/STROKEAHA.111.640995.

4. Dolan JM, Kolega J, Meng H. High wall shear stress and spatial gradients in vascular pathology: a review. Annals of biomedical engineering 2013; 41(7):1411-1427, doi:10.1007/s10439-012-0695-0.

5. Kulcsár Z, Ugron A, Marosfói M, Berentei Z, Paál G, Szikora I. Hemodynamics of cerebral aneurysm initiation: the role of wall shear stress and spatial wall shear stress gradient. American Journal of Neuroradiology 2011; 32(3):587-594, doi:10.3174/ajnr.A2339.

6. Samady H, Eshtehardi P, McDaniel MC, Suo J, Dhawan SS, Maynard C, Timmins LH, Quyyumi AA, Giddens DP. Coronary artery wall shear stress is associated with progression and transformation of atherosclerotic plaque and arterial remodeling in patients with coronary artery disease. Circulation 2011; 124(7):779-788, doi: 10.1161/CIRCULATIONAHA.111.021824.

7. Johnston BM, Johnston PR, Corney S, Kilpatrick D. Non-Newtonian blood flow in human right coronary arteries: transient simulations. Journal of biomechanics 2006; 39(6):1116-1128.

8. Gambaruto A, Janela J, Moura A, Sequeira A. Shear-thinning effects of hemodynamics in patient-specific cerebral aneurysms. Mathematical biosciences and engineering 2013; 10(3):649-665.

9. Myers JG, Moore JA, Ojha M, Johnston KW, Ethier CR. Factors influencing blood flow patterns in the human right coronary artery. Annals of Biomedical Engineering 2001; 29(2):109-120, doi:10.1114/1.1349703.

10. Ramalho S, Moura A, Gambaruto AM, Sequeira A. Sensitivity to outflow boundary conditions and level of geometry description for a cerebral aneurysm. International Journal for Numerical Methods in Biomedical Engineering 2012; 28(6-7):697-713, doi:10.1002/cnm.2461.

11. Cebral JR, Castro MA, Appanaboyina S, Putman CM, Millan D, Frangi AF. Efficient pipeline for image-based patient-specific analysis of cerebral aneurysm hemodynamics: technique and sensitivity. IEEE transactions on medical imaging 2005; 24(4):457-467.

12. Gambaruto A, Doorly D, Yamaguchi T. Wall shear stress and near-wall convective transport: Comparisons with vascular remodelling in a peripheral graft anastomosis. Journal of Computational Physics 2010; 229(14):5339 5356, doi:10.1016/j.jcp.2010.03.029.

13. Gambaruto AM, Peiró J, Doorly DJ, Radaelli AG. Reconstruction of shape and its effect on flow in arterial conduits. International Journal for Numerical Methods in Fluids 2008; 57(5):495-517, doi:10.1002/fld.1642.

14. Ramalho S, Moura A, Gambaruto AM, Sequeira A. Influence of blood rheology and outflow boundary conditions in numerical simulation of cerebral aneursyms. Springer, 2013; 149-175, doi:10.1007/978-1-4614-4178-6_6.

15. Jiang J, Johnson K, Valen-Sendstad K, Mardal KA, Wieben O, Strother C. Flow characteristics in a canine aneurysm model: a comparison of 4D accelerated phase-contrast MR measurements and computational fluid dynamics simulations. Medical physics 2011; 38(11):6300-6312, doi:10.1118/1.3652917.

16. Jain K, Jiang J, Strother C, Mardal KA. Transitional hemodynamics in intracranial aneurysms-comparative velocity investigations with high resolution lattice Boltzmann simulations, normal resolution ANSYS simulations, and MR imaging. Medical Physics 2016; 43(11):6186-6198.

17. Evju Ø, Pozo JM, Frangi A, Mardal KA. Robustness of common hemodynamics indicators with respect to numerical resolution in 38 middle cerebral artery aneurysms. PLOS ONE 2017;

18. Valen-Sendstad K, Mardal KA, Mortensen M, Reif BAP, Langtangen HP. Direct numerical simulation of transitional flow in a patient-specific intracranial aneurysm. Journal of biomechanics 2011; 44(16):2826-2832.

19. Valen-Sendstad K, Steinman D. Mind the gap: impact of computational fluid dynamics solution strategy on prediction of intracranial aneurysm hemodynamics and rupture status indicators. American Journal of Neuroradiology 2014; 35(3):536-543.

20. Funamoto K, Suzuki Y, Hayase T, Kosugi T, Isoda H. Numerical validation of MR-measurement-integrated simulation of blood flow in a cerebral aneurysm. Annals of biomedical engineering 2009; 37(6):1105-1116.

21. Bertagna L, D’Elia M, Perego M, Veneziani A. Data assimilation in cardiovascular fluid-structure interaction problems: an introduction. Fluid-Structure Interaction and Biomedical Applications. Springer, 2014; 395-481.

22. Fursikov AV, Gunzburger MD, Hou LS. Boundary value problems and optimal boundary control for the NavierStokes system: the two-dimensional case. SIAM Journal on Control and Optimization 1998; 36(3):852-894, doi: 10.1137/S0363012994273374.

23. Lee H. Optimal control for quasi-Newtonian flows with defective boundary conditions. Computer Methods in Applied Mechanics and Engineering 2011; 200(33):2498-2506, doi:10.1016/j.cma.2011.04.019.

24. Guerra T, Sequeira A, Tiago J. Existence of optimal boundary control for the Navier-Stokes equations with mixed boundary conditions. Portugaliae Mathematica 2015; 72:267-283, doi:10.4171/pm/1968.

25. Guerra T, Catarino C, Mestre T, Santos S, Tiago J, Sequeira A. A data assimilation approach for non-Newtonian blood flow simulations in 3D geometries. Applied Mathematics and Computation 2018; 321:176 - 194, doi: 10.1016/j.amc.2017.10.029.

26. D’Elia M, Mirabella L, Passerini T, Perego M, Piccinelli M, Vergara C, Veneziani A. Applications of variational data assimilation in computational hemodynamics. Springer Milan, 2012; 363-394, doi:10.1007/ 978-88-470-1935-5_12.

27. D'Elia M, Perego M, Veneziani A. A variational data assimilation procedure for the incompressible NavierStokes equations in hemodynamics. Journal of Scientific Computing 2012; 52(2):340-359, doi:10.1007/ s10915-011-9547-6. 
28. John LJ. Optimal Boundary Control in Energy Spaces, vol. 24. Verlag der Technischen Universität Graz, 2014, doi:10.3217/978-3-85125-373-3.

29. Tiago J, Gambaruto A, Sequeira A. Patient-specific blood flow simulations: Setting Dirichlet boundary conditions for minimal error with respect to measured data. Mathematical Modelling of Natural Phenomena 2014; 9(06):98116, doi:10.1051/mmnp/20149608.

30. Perego M, Veneziani A, Vergara C. A variational approach for estimating the compliance of the cardiovascular tissue: An inverse fluid-structure interaction problem. SIAM Journal on Scientific Computing 2011; 33(3):11811211.

31. Dwight RP. Bayesian inference for data assimilation using least-squares finite element methods. IOP Conference Series: Materials Science and Engineering, vol. 10, IOP Publishing, 2010; 012224.

32. Lassila T, Manzoni A, Quarteroni A, Rozza G. A reduced computational and geometrical framework for inverse problems in hemodynamics. International journal for numerical methods in biomedical engineering 2013; 29(7):741-776.

33. Manzoni A, Lassila T, Quarteroni A, Rozza G. A reduced-order strategy for solving inverse Bayesian shape identification problems in physiological flows. Modeling, Simulation and Optimization of Complex ProcessesHPSC 2012. Springer, 2014; 145-155.

34. Quarteroni A, Rozza G. Optimal control and shape optimization of aorto-coronaric bypass anastomoses. Mathematical Models and Methods in Applied Sciences 2003; 13(12):1801-1823.

35. Heys JJ, Manteuffel TA, McCormick SF, Milano M, Westerdale J, Belohlavek M. Weighted least-squares finite elements based on particle imaging velocimetry data. Journal of Computational Physics 2010; 229(1):107-118.

36. Formaggia L, Veneziani A, Vergara C. A new approach to numerical solution of defective boundary value problems in incompressible fluid dynamics. SIAM Journal on Numerical Analysis 2008; 46(6):2769-2794.

37. Veneziani A, Vergara C. An approximate method for solving incompressible Navier-Stokes problems with flow rate conditions. Computer methods in applied mechanics and engineering 2007; 196(9):1685-1700.

38. Mohammadi B, Pironneau O. Applied shape optimization for fluids. Oxford university press, 2010.

39. Steinman DA. Assumptions in modelling of large artery hemodynamics. Modeling of Physiological Flows. Springer, 2012; 1-18, doi:10.1007/978-88-470-1935-5_1.

40. Evju Ø, Valen-Sendstad K, Mardal KA. A study of wall shear stress in 12 aneurysms with respect to different viscosity models and flow conditions. Journal of biomechanics 2013; 46(16):2802-2808, doi:10.1016/j.jbiomech. 2013.09.004.

41. Guerra T, Tiago J, Sequeira A. Optimal control in blood flow simulations. International Journal of Non-Linear Mechanics 2014; 64:57-69.

42. Le Dimet FX, Talagrand O. Variational algorithms for analysis and assimilation of meteorological observations: theoretical aspects. Tellus A: Dynamic Meteorology and Oceanography 1986; 38(2):97-110.

43. Bertino L, Evensen G, Wackernagel H. Sequential data assimilation techniques in oceanography. International Statistical Review 2003; 71(2):223-241.

44. Bunge HP, Hagelberg C, Travis B. Mantle circulation models with variational data assimilation: inferring past mantle flow and structure from plate motion histories and seismic tomography. Geophysical Journal International 2003; 152(2):280-301.

45. Talagrand O. Variational Assimilation. Springer Berlin Heidelberg: Berlin, Heidelberg, 2010; 41-67, doi:10.1007/ 978-3-540-74703-1_3.

46. Hinze M, Pinnau R, Ulbrich M, Ulbrich S. Optimization with PDE constraints, vol. 23. Springer Science \& Business Media, 2008.

47. Nocedal J, Wright SJ. Numerical Optimization. Springer Science \& Business Media, 2006, doi:10.1007/ 978-0-387-40065-5.

48. Schwedes T, Funke SW, Ham DA. An iteration count estimate for a mesh-dependent steepest descent method based on finite elements and Riesz inner product representation 2016; .

49. Kelley CT, Sachs EW. Quasi-Newton methods and unconstrained optimal control problems. SIAM Journal on Control and Optimization 1987; 25(6):1503-1516, doi:10.1137/0325083.

50. Hager WW. Runge-kutta methods in optimal control and the transformed adjoint system. Numerische Mathematik 2000; 87(2):247-282, doi:10.1007/s002110000178.

51. Sandu A. On the Properties of Runge-Kutta Discrete Adjoints. Springer Berlin Heidelberg: Berlin, Heidelberg, 2006; 550-557, doi:10.1007/11758549 76.

52. Walther A. Automatic differentiation of explicit runge-kutta methods for optimal control. Computational Optimization and Applications 2007; 36(1):83-108, doi:10.1007/s10589-006-0397-3.

53. Nitsche J. Über ein Variationsprinzip zur Lösung von Dirichlet-Problemen bei Verwendung von Teilräumen, die keinen Randbedingungen unterworfen sind. Abhandlungen aus dem mathematischen Seminar der Universität Hamburg, vol. 36, Springer, 1971; 9-15, doi:10.1007/BF02995904.

54. Becker R, Burman E, Hansbo P. A Nitsche extended finite element method for incompressible elasticity with discontinuous modulus of elasticity. Computer Methods in Applied Mechanics and Engineering 2009; 198(41):3352-3360, doi:10.1016/j.cma.2009.06.017.

55. Burman E, Hansbo P. A unified stabilized method for Stokes' and Darcy's equations. Journal of Computational and Applied Mathematics 2007; 198(1):35-51, doi:10.1016/j.cam.2005.11.022.

56. Gunzburger M. Perspectives in Flow Control and Optimization. Society for Industrial and Applied Mathematics, 2002, doi:10.1137/1.9780898718720.

57. Logg A, Mardal KA, Wells GN. Automated Solution of Differential Equations by the Finite Element Method. Springer, 2012, doi:10.1007/978-3-642-23099-8.

58. Farrell PE, Ham DA, Funke SW, Rognes ME. Automated derivation of the adjoint of high-level transient finite element programs. SIAM Journal on Scientific Computing 2013; 35(4):C369-C393, doi:10.1137/120873558.

59. Morozov VA. On the solution of functional equations by the method of regularization. Soviet Math. Dokl, vol. 7, 1966; 414-417. 
60. Hansen PC. Rank-deficient and discrete ill-posed problems: numerical aspects of linear inversion, vol. 4. Siam, 1998, doi:10.1137/1.9780898719697.

61. Bonesky T. Morozov's discrepancy principle and Tikhonov-type functionals. Inverse Problems 2009; 25(1):015 015, doi:10.1088/0266-5611/25/1/015015.

62. Eberhard P, Bischof C. Automatic differentiation of numerical integration algorithms. Mathematics of Computation of the American Mathematical Society 1999; 68(226):717-731.

63. Alexe M, Sandu A. On the discrete adjoints of adaptive time stepping algorithms. Journal of Computational and Applied Mathematics 2009; 233(4):1005 - 1020, doi:10.1016/j.cam.2009.08.109.

64. Herzog R, Kunisch K. Algorithms for pde-constrained optimization. GAMM-Mitteilungen 2010; 33(2):163-176, doi:10.1002/gamm.201010013.

65. Clever D, Lang J, Ulbrich S, Ziems JC. Combination of an adaptive multilevel SQP method and a space-time adaptive pdae solver for optimal control problems. Procedia Computer Science 2010; 1(1):1435 - 1443, doi: 10.1016/j.procs.2010.04.159. 